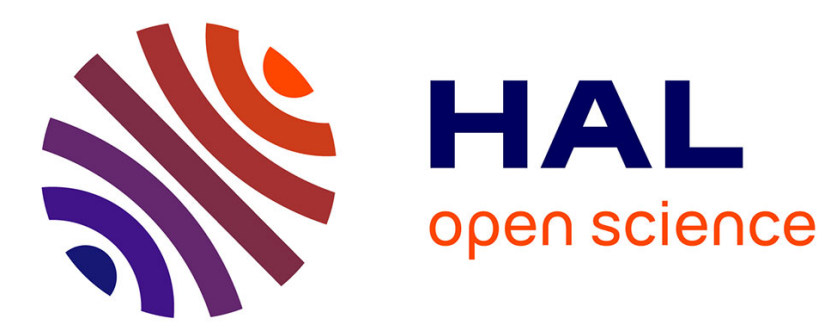

\title{
Natural doubly diffusive convection with vibration
}

Gérald Bardan, Edgar Knobloch, Abdelkader Mojtabi, Hicham Khallouf

\section{To cite this version:}

Gérald Bardan, Edgar Knobloch, Abdelkader Mojtabi, Hicham Khallouf. Natural doubly diffusive convection with vibration. Fluid Dynamics Research, 2000, 28 (3), pp.159-187. 10.1016/S01695983(00)00028-9 . hal-01838576

\section{HAL Id: hal-01838576 https://hal.science/hal-01838576}

Submitted on 13 Jul 2018

HAL is a multi-disciplinary open access archive for the deposit and dissemination of scientific research documents, whether they are published or not. The documents may come from teaching and research institutions in France or abroad, or from public or private research centers.
L'archive ouverte pluridisciplinaire HAL, est destinée au dépôt et à la diffusion de documents scientifiques de niveau recherche, publiés ou non, émanant des établissements d'enseignement et de recherche français ou étrangers, des laboratoires publics ou privés. 


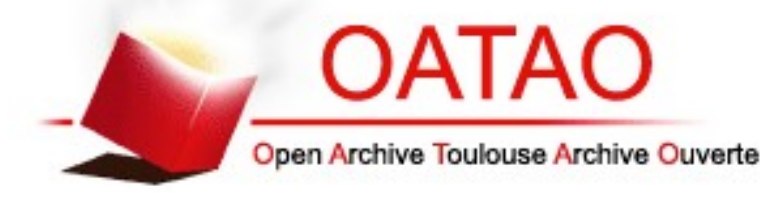

Open Archive Toulouse Archive Ouverte

OATAO is an open access repository that collects the work of Toulouse researchers and makes it freely available over the web where possible

This is an author's version published in: http://oatao.univ-toulouse.fr/20615

\section{Official URL:}

http://dx.doi.org/10.1016/S0169-5983(00)00028-9

\section{To cite this version:}

Bardan, Gérald and Knobloch, Edgar and Mojtabi, Abdelkader and Khallouf, Hicham Natural doubly diffusive convection with vibration. (2000) Fluid Dynamics Research, 29. pp. 159 - 187. ISSN 0169-5983

Any correspondence concerning this service should be sent to the repository administrator: tech-oatao@listes-diff.inp-toulouse.fr 


\title{
Natural doubly diffusive convection with vibration
}

\author{
G. Bardan ${ }^{\mathrm{a}, *}$, E. Knobloch ${ }^{\mathrm{b}}$, A. Mojtabi ${ }^{\mathrm{a}}$, H. Khallouf ${ }^{\mathrm{c}}$ \\ ${ }^{a}$ U.M.R. 5502 IMFT-CNRS-UPS, U.F.R. M.I.G., Universite Paul Sabatier, 118, route de Narbonne, \\ 31062 Toulouse Cedex, France \\ ${ }^{\mathrm{b}}$ Department of Physics, University of California, Berkeley, CA 94720, USA \\ ${ }^{\mathrm{c} M e d y s y s,} 29$ rue Jean Rostand, 91893 Orsay Cedex, France
}

\begin{abstract}
We present a numerical and analytical study of doubly diffusive convection driven by horizontal thermal and solutal gradients in square and rectangular enclosures with no-slip walls subjected to high-frequency vibration. The two vertical walls of the enclosure are maintained at different but uniform temperatures and concentrations while the horizontal walls are assumed to be impermeable and insulating. The resulting system is described by time-averaged Boussinesq equations. These equations possess a doubly diffusive quasi-equilibrium solution provided the thermal and solutal buoyancy forces are equal and opposite. This solution is linearly stable up to a critical value of the stability parameter independently of the strength and orientation of the vibration. The solutions in the neighborhood of the bifurcation point are described analytically as a function of the strength and orientation of the vibration, and the larger amplitude states are computed numerically using a spectral collocation method. For vertical oscillation increasing the vibration amplitude decreases the subcriticality of the solutions and may even reverse it; the opposite occurs with horizontal vibration. (c) 2001 Published by The Japan Society of Fluid Mechanics and Elsevier Science B.V. All rights reserved.
\end{abstract}

Keywords: Doubly diffusive convection; Vibration and bifurcation

\section{Introduction}

Many convective phenomena involve both heat and mass transfer. Doubly diffusive processes in enclosures, such as those arising in geology or in chemical applications, serve as a generic model of such systems. A review of doubly diffusive convection in these systems is given by Viskanta et al. (1985). Recently, doubly diffusive convection in enclosures has received increased attention since it is closely related to transfer processes in crystal growth (Wilcox, 1993).

In the present work we restrict our attention to a fluid layer subjected to horizontal temperature and concentration gradients with the property that the thermal and concentration buoyancy forces

* Corresponding author. Tel.: +33-5-61-55-6788; fax: +33-0-5-61-55-8326.

E-mail address: bardan@imft.fr (G. Bardan). 
exactly balance. In this case there exists an exact equilibrium solution with zero velocity and linear temperature and concentration profiles, and instability is caused by the difference between the solutal and thermal diffusivities, measured by the Lewis number Le. When Le $=1$ the diffusive solution is stable for all Rayleigh numbers. When Le $\neq 1$ there is a critical value of the Rayleigh number at which this solution undergoes a bifurcation and above which motion is observed. The square cavity case was previously investigated by Krishnan (1989) for $\operatorname{Pr}=1$ and Le $=3.161$ whose numerical study indicated that the onset of convection occurs at a critical thermal Rayleigh number $\operatorname{Ra}_{T_{\text {conv }}}=3000\left(\operatorname{Ra}_{T_{\text {conv }}}(\mathrm{Le}-1)=6481\right)$. Below this point the purely diffusive solution is stable. Gobin and Bennacer (1994) have studied theoretically the case of an infinite vertical layer with free-slip boundary conditions and showed that the critical Rayleigh number obeys the relation $\operatorname{Ra}_{T_{\mathrm{c}}}(\mathrm{Le}-1)=$ 6122. With no-slip boundary conditions the corresponding result is $\operatorname{Ra}_{T_{\mathrm{c}}}(\mathrm{Le}-1)=17172$ (Ghorhayeb and Mojtabi, 1997). These results indicate that the lowest value of the convective threshold reported by Krishnan (1989) in fact corresponds to a turning point on a finite amplitude subcritical branch (Gobin and Bennacer, 1994). The full solution branch was constructed using Newton's method, combined with a continuation scheme, by Xin et al. (1998). The subcritical branch corresponds to a clockwise cell while the supercritical branch corresponds to a three-cell solution consisting of a large counterclockwise cell slanted across the cavity with smaller clockwise cells located in opposite corners. Mechanisms responsible for oscillatory doubly diffusive convection have been discussed by Chang and Lin (1993) and by Alavyoon (1994).

A possible application of interest arises in materials processing under reduced gravity conditions when convection due to buoyancy forces is strongly reduced. In zero gravity the desired basic state may be set up in a melt when the temperature and concentration gradients are parallel. However, any residual gravity and in particular fluctuations in the effective gravity due to orientation changes of the vehicle and on-board activities introduce significant perturbations. As a rule, the residual acceleration fields on board of a spacecraft are non-stationary and the measured oscillation frequencies range from $10^{-2} \mathrm{~Hz}$ to 10 or $100 \mathrm{~Hz}$ (Belyaev et al., 1994 and Rogers and DeLombard, 1995). Crew activity or orbital manoeuvres give rise to time-dependent accelerations ( $g$-jitter) with amplitudes as high as $10^{-4}$ to $10^{-3} \mathrm{~g}$ and fluctuating direction. The literature on the effects of g-jitter on materials processing is quite extensive; for a recent comprehensive review the reader is referred to Nelson (1991). A recent review by Alexander (1990) summarizes the important concerns and discusses the effect of residual accelerations on heat and mass transfer in low-gravity materials experiments. Alexander et al. (1991) also analyze low-gravity-tolerance (or Bridgman-Stockbarger) crystal growth. The effect of gravity modulation on the onset of convection for unidirectional solidification was considered by Murray et al. (1991) while Wheeler et al. (1991) analyzed the high-frequency limit of this problem. In this limit the method of averaging may be used to determine the effect of large-amplitude gravity modulation on both the primary instability and its subsequent evolution. In contrast, low-frequency $(<1 \mathrm{~Hz})$ sinusoidal modulation has to be treated via Floquet theory.

Full Navier-Stokes simulations of the Bénard problem for a one-component fluid in a finite box carried out by Birigen and Peltier (1990) agree with the stability calculations of Gresho and Sani (1970). The effect of vibration on the onset of convection in a horizontally unbounded two-component fluid has also been studied. Gershuni and Zhukhovitsky (1979) computed the flow in an enclosed differentially heated cavity at $\mathrm{Ra}=10^{4}$ for finite frequencies and various values of the vibrational Rayleigh number and showed that a strong resonance can take place that increases the heat flux by more than a factor of two. The resonance frequency depends on both the Rayleigh number and the 
amplitude of the vibration. Finally, Terrones and Chen (1993) describe the effect of time-dependent sinusoidal gravity perpendicular to the boundaries on the onset of convection in a horizontal doubly cross-diffusive fluid layer. The topology of the neutral curves is more complex than that encountered in constant-gravity multiply diffusive layers, leading to new types of behavior not possible in the absence of modulation.

The averaging method, suitable for high-frequency ( $>1 \mathrm{~Hz}$ ) modulation, was first used for thermovibrational convection by Simonenko and Zen'kovskaja (1966) and this is the approach adopted in the present paper. Using the averaged equations (Gershuni and Zhukhovitsky, 1979, 1981, 1988) performed linear stability analyses for a fluid layer with various boundary conditions. These authors introduced the vibrational analog of the Rayleigh number $R_{\mathrm{v}}$ to represent the strength of the vibration source, and studied the Rayleigh-Bénard problem as a function of the vibration angle, and the interaction between natural and thermo-vibrational convection. More recent motivation for extending this work has been provided by the development of space experiments and the use of mechanical vibration in industrial processes requiring control of convective motions. In addition to the stability analysis, numerical simulations of closed cavities, either heated from below or from the side, have also been done (Chernatynsky et al., 1995; Khallouf et al., 1995). For binary mixtures Braverman (1987) considered the case when the thermal and solutal gradients and the vibration axis are parallel and perpendicular to the layer; a solution describing a long wave mode was obtained. More recently, Gershuni et al. (1997) analyzed the linear stability of a binary mixture in a plane horizontal layer with the Soret effect subject to static gravity and a longitudinal high-frequency vibration, focusing on long-wave modes. Situations involving both instability mechanisms, gravitational and vibrational, were studied numerically for representative values of the parameters. Three types of instability can be distinguished: monotonic cellular, monotonic long wave and oscillatory cellular. In the case of the normal Soret effect only the monotonic instability takes place and thermodiffusion plays a destabilizing role; in contrast, in the case of the anomalous Soret effect the vibrational instability depends strongly on the Soret parameter. To the best of our knowledge, no papers have appeared on the corresponding problem in enclosures.

In this paper we present an extensive discussion of the effect of high-frequency vibration on doubly diffusive convection in square and rectangular enclosures. We describe the results of a numerical study of the influence of the direction of the vibration axis on thermosolutal convection in cavities bounded by two vertical walls maintained at different but uniform temperatures and concentrations making equal and opposite contributions to the net buoyancy force. Since the main purpose of conducting experiments in space lies in eliminating or significantly decreasing natural convection, it is important to understand the influence of any residual acceleration on the convection amplitude. In Section 2 we summarize the mathematical model and the time-averaged form of the Boussinesq equations. In Section 3 we show that the onset of convection is unaffected by the presence of vibration. This is not so, however, for the nonlinear problem and we describe in Section 4 the effects of vibration on the weakly nonlinear regime. In square cavities convection sets in via a transcritical bifurcation with the subcritical branch turning towards higher Rayleigh numbers at a saddle-node bifurcation. For larger aspect ratios the primary bifurcation may be a (subcritical) pitchfork. In either case the subcritical solutions acquire stability at a secondary saddle-node bifurcation; the location of this turning point is affected by vibration leading to the possibility of control of the onset of finite amplitude convection. These results are described in Section 5. The paper concludes with a brief discussion in Section 6. 


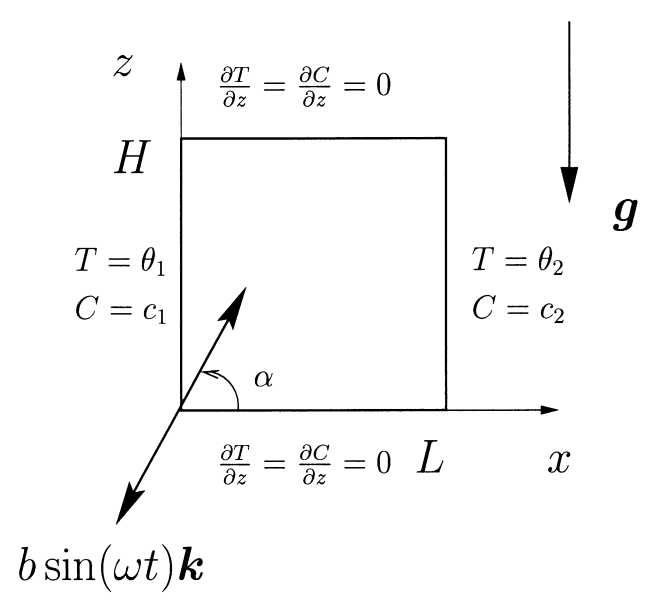

Fig. 1. Sketch of the cavity configuration.

\section{Problem description and basic equations}

The system considered is two-dimensional thermosolutal convection in a container. Fig. 1 shows the flow configuration and coordinate system. The flow domain is $(x, z) \in \Omega=[0, L] \times[0, H]$. All the physical properties are taken to be constant. The vertical walls at $x=0$ and $L$ are kept at constant and uniform temperatures $\theta_{1}$ and $\theta_{2}$, respectively, and at constant and uniform concentrations $c_{1}$ and $c_{2}$, with $\theta_{1}>\theta_{2}$ and $c_{1}>c_{2}$. The top (at $z=H$ ) and bottom (at $z=0$ ) horizontal walls are insulated. All the boundaries are assumed rigid and the horizontal ones impermeable. The fluid cavity with its boundaries is subject to linear harmonic oscillations.

The binary fluid in the cavity is considered to be Newtonian and to satisfy the Boussinesq approximation. The Dufour and Soret effects are neglected. The thermophysical properties are constant except for the fluid density in the buoyancy term which depends linearly on the local temperature and concentration. The equation of state has the form

$$
\rho(\theta, c)=\rho_{\text {ref }}\left(1-\beta_{T}\left(\theta-\theta_{\text {ref }}\right)-\beta_{C}\left(c-c_{\text {ref }}\right)\right),
$$

where $\rho_{\text {ref }}=\rho\left(\theta_{\text {ref }}, c_{\text {ref }}\right), \beta_{T}=-1 / \rho_{\text {ref }}(\partial \rho / \partial \theta)_{c}$ and $\beta_{C}=-1 / \rho_{\text {ref }}(\partial \rho / \partial c)_{\theta}$ are, respectively, the density at the standard temperature $\theta_{\text {ref }}=\theta_{2}$ and the standard concentration $c_{\text {ref }}=c_{2}$, and the thermal and solutal expansion coefficients. The quantity $\beta_{T}$ is positive and, if $c$ denotes the concentration of the heavier component, $\beta_{C}$ is negative. As a result the thermal contribution to the buoyancy force favors a clockwise circulation while the solutal contribution promotes counterclockwise circulation.

The presence of vibration changes the gravitational acceleration according to

$$
\boldsymbol{g} \rightarrow \boldsymbol{g}-b \omega^{2} \sin (\omega t) \boldsymbol{k},
$$

where $\boldsymbol{k}=\cos \alpha \boldsymbol{x}+\sin \alpha \boldsymbol{z}$ is the unit vector along the vibration axis and $\alpha=(\boldsymbol{x}, \boldsymbol{k})$ is the vibration angle, $b$ is the displacement amplitude and $\omega$ is the angular frequency. Using the velocity $\boldsymbol{u}$, the pressure $p$, the temperature $\theta$ and the concentration $c$ as independent variables, the system is then 
described by the nondimensional primitive equations

$$
\begin{aligned}
& \nabla \cdot \boldsymbol{u}=0, \\
& \frac{\partial \boldsymbol{u}}{\partial t}+(\boldsymbol{u} \cdot \nabla) \boldsymbol{u}=-\nabla p+\nabla^{2} \boldsymbol{u}+\left(\mathrm{Gr}_{T} \theta+\mathrm{Gr}_{C} c\right) \boldsymbol{z}+\left(\mathrm{Gr}_{T V} \theta+\mathrm{Gr}_{C V} c\right) \hat{\omega} \sin (\hat{\omega} t) \boldsymbol{k}, \\
& \frac{\partial \theta}{\partial t}+(\boldsymbol{u} \cdot \nabla) \theta=\frac{1}{\operatorname{Pr}} \nabla^{2} \theta, \\
& \frac{\partial c}{\partial t}+(\boldsymbol{u} \cdot \nabla) c=\frac{1}{\mathrm{Sc}} \nabla^{2} c .
\end{aligned}
$$

These equations have been nondimensionalized with respect to the viscous diffusion time in the horizontal using $L$ as the lengthscale. Velocities are expressed in terms of $v / L$, where $v$ is the kinematic diffusivity, while the temperature and concentration differences from their reference values $\theta_{2}$ and $c_{2}$ are nondimensionalized using $\theta_{1}-\theta_{2}$ and $c_{1}-c_{2}$, respectively. The nondimensional boundary conditions are therefore

$$
\begin{aligned}
& \boldsymbol{u}=\mathbf{0} \quad \text { on } \partial \Omega, \\
& \theta=1, \quad c=1 \quad \text { for } x=0, \forall z, \\
& \theta=0, \quad c=0 \quad \text { for } x=1, \forall z, \\
& \frac{\partial \theta}{\partial z}=\frac{\partial c}{\partial z}=0 \quad \text { for } z=0 \quad \text { and } \quad A, \forall x .
\end{aligned}
$$

The problem is specified by seven nondimensional parameters: the thermal Grashof number $\mathrm{Gr}_{T}=$ $g \beta_{T}\left(\theta_{1}-\theta_{2}\right) L^{3} / v^{2}$, the solutal Grashof number $\operatorname{Gr}_{C}=g \beta_{C}\left(c_{1}-c_{2}\right) L^{3} / v^{2}$, the modified vibrational thermal Grashof number $\operatorname{Gr}_{T V}=b \omega \beta_{T}\left(\theta_{1}-\theta_{2}\right) L / v$, the modified vibrational solutal Grashof number $\mathrm{Gr}_{C V}=b \omega \beta_{C}\left(c_{1}-c_{2}\right) L / v$, the dimensionless vibration frequency $\hat{\omega}=\omega\left(L^{2} / v\right)$, the Prandtl number $\operatorname{Pr}=v / a$ ( $a$ is the thermal diffusivity), the Schmidt number $\operatorname{Sc}=v / D$ ( $D$ is the mass diffusivity) and the aspect ratio $A=H / L$. The Lewis number is defined to be $\mathrm{Le}=\mathrm{Sc} / \mathrm{Pr}$. The modified vibrational Grashof numbers (thermal or solutal) are obtained by replacing $g$ by $b \omega^{2}$ in the Grashof number and rescaling by the ratio between the viscous diffusive time and the vibrational time $\omega L^{2} / v$. In the limit of high frequency and small amplitude the effect of vibration is determined by the product $b \omega$ appearing in the definition of the modified vibrational Grashof numbers $\mathrm{Gr}_{T V}$ and $\mathrm{Gr}_{C V}$. In the following we use the Grashof numbers as parameters of the problem, instead of the Rayleigh numbers; these are related by the simple relation $\mathrm{Ra}_{T}=\mathrm{Gr}_{T} \mathrm{Pr}$.

In the asymptotic case of high-frequency small amplitude vibration the method of averaging can be applied to obtain a closed system of equations for the slow evolution of the mean velocity $\boldsymbol{U}$, mean temperature $T$, mean concentration $C$ and mean pressure $P$. The method is analogous to that employed by Schlichting (1932) to compute the boundary condition on inviscid flow in the bulk due to the presence of an oscillatory viscous boundary layer, and results in the following set of 
equations (Landau and Lifshitz, 1988; Gershuni and Lyubimov, 1998)

$$
\begin{aligned}
& \nabla \cdot \boldsymbol{U}=0, \\
& \frac{\partial \boldsymbol{U}}{\partial t}+(\boldsymbol{U} . \nabla) \boldsymbol{U}=-\nabla P+\nabla^{2} \boldsymbol{U}+\mathrm{Gr}_{T}(T+N C) \boldsymbol{z} \\
& +\frac{1}{2} \mathrm{Gr}_{T V}^{2}\left(\boldsymbol{W}_{T}+N \boldsymbol{W}_{c}\right) \cdot \nabla\left(T \boldsymbol{k}-\boldsymbol{W}_{T}+N\left(C \boldsymbol{k}-\boldsymbol{W}_{c}\right)\right), \\
& \frac{\partial T}{\partial t}+(\boldsymbol{U} \cdot \nabla) T=\frac{1}{\operatorname{Pr}} \nabla^{2} T, \\
& \frac{\partial C}{\partial t}+(\boldsymbol{U} . \nabla) C=\frac{1}{\mathrm{Sc}} \nabla^{2} C, \\
& \nabla \cdot \boldsymbol{W}_{T}=0, \quad \operatorname{curl}\left(\boldsymbol{W}_{T}\right)=\operatorname{curl}(T \boldsymbol{k}), \\
& \nabla \cdot \boldsymbol{W}_{c}=0, \quad \operatorname{curl}\left(\boldsymbol{W}_{c}\right)=\operatorname{curl}(C \boldsymbol{k}),
\end{aligned}
$$

where $N \equiv \mathrm{Gr}_{C} / \mathrm{Gr}_{T}=\mathrm{Gr}_{C V} / \mathrm{Gr}_{T V}$. These equations describe the evolution of the mean fields on a timescale much longer than the vibration period $\tau=2 \pi / \omega$; the new term in Eq. (12) is the consequence of the quadratic nonlinearity in the Navier-Stokes equation. The quantities $\boldsymbol{W}_{T}$ and $\boldsymbol{W}_{c}$ represent the solenoidal parts of the vectors $T \boldsymbol{k}$ and $C \boldsymbol{k}$, respectively, and also vary slowly with time. In terms of these quantities the fluctuating velocity field $\boldsymbol{u}^{\prime}$ is given by $\boldsymbol{u}^{\prime}=-\mathrm{Gr}_{T V} \cos \omega t \boldsymbol{W}_{T}-\mathrm{Gr}_{C V} \cos \omega t \boldsymbol{W}_{c}$. Eqs. (15), (16) are to be solved subject to the boundary conditions (7)-(10) together with

$$
\boldsymbol{W}_{T} \cdot \boldsymbol{n}=\boldsymbol{W}_{c} \cdot \boldsymbol{n}=0 \quad \text { on } \partial \Omega,
$$

where $\boldsymbol{n}$ is the unit outward normal.

The averaged equations are valid under the assumptions: (i) the frequency must be sufficiently high (but not acoustic) that the vibration period is small compared to all the characteristic hydrodynamic times $\tau \ll \min \left(L^{2} / a, L^{2} / D, L^{2} / v\right)$, (ii) the displacement amplitude must be small in the sense that $b \ll \min \left(L / \beta_{T}\left(\theta_{1}-\theta_{2}\right), L / \beta_{C}\left(c_{1}-c_{2}\right)\right)$. Simonenko (1972) proves the convergence of solutions of the averaged system to averaged solutions of the system (3)-(6). A numerical description of this convergence is given by Khallouf (1995), while Gershuni and Lyubimov (1998) show that the new terms act as a vibrational body force directed opposite to the kinetic energy gradient.

\section{Linear stability}

In the following we write $R_{\mathrm{v}} \equiv \frac{1}{2} \mathrm{Gr}_{T V}^{2}$ and refer to it as the vibrational Grashof number. The thermal Grashof number $\mathrm{Gr}_{T}$ will be written as $\mathrm{Gr}$.

\subsection{Mechanical equilibrium and its stability}

We refer to states with $\boldsymbol{U}=\mathbf{0}$ as a mechanical quasi-equilibrium. Such states have zero mean velocity but the fluctuating velocity does not necessarily vanish. In order for a quasi-equilibrium to 
exist the following equations must be satisfied:

$$
\begin{aligned}
& \operatorname{Gr}\left(T_{0}+N C_{0}\right) \boldsymbol{z}+R_{\mathrm{v}}\left(\boldsymbol{W}_{T_{0}}+N \boldsymbol{W}_{c_{0}}\right) \cdot \nabla\left(T_{0} \boldsymbol{k}-\boldsymbol{W}_{T_{0}}+N\left(C_{0} \boldsymbol{k}-\boldsymbol{W}_{c_{0}}\right)\right)=\nabla P_{0}, \\
& \Delta T_{0}=0, \\
& \Delta C_{0}=0, \\
& \nabla \cdot \boldsymbol{W}_{T_{0}}=0, \quad \operatorname{curl} \boldsymbol{W}_{T_{0}}=\operatorname{curl}\left(T_{0} \boldsymbol{k}\right), \\
& \nabla \cdot \boldsymbol{W}_{c_{0}}=0, \quad \operatorname{curl} \boldsymbol{W}_{c_{0}}=\operatorname{curl}\left(C_{0} \boldsymbol{k}\right)
\end{aligned}
$$

subject to the boundary conditions (7)-(10) and (17). Eqs. (19) and (20) and the uniqueness of the Helmholtz decomposition lead to the solution $T_{0}=C_{0}=1-x$ and hence to $\boldsymbol{W}_{T_{0}}=\boldsymbol{W}_{c_{0}}$. Eq. (18) now becomes

$$
\operatorname{Gr}(1+N) T_{0} \boldsymbol{z}+R_{\mathrm{v}}(1+N)^{2} \boldsymbol{W}_{T_{0}} . \nabla\left(T_{0} \boldsymbol{k}-\boldsymbol{W}_{T_{0}}\right)=\nabla P_{0} .
$$

One can check that this equality holds if and only if $N=-1$. This assumption implies that the contributions to the buoyancy force from the thermal and solutal gradients exactly cancel. Two cases are of particular interest:

- Horizontal vibration. In this case $\boldsymbol{k}=\boldsymbol{x}$ and $\boldsymbol{W}_{T_{0}}=\boldsymbol{W}_{c_{0}}=\mathbf{0}$. Consequently in this case we have a true mechanical equilibrium, i.e., the oscillatory components of the velocity also vanish.

- Vertical vibration. In this case $\boldsymbol{k}=\boldsymbol{z}$ and $\boldsymbol{W}_{T_{0}}=\boldsymbol{W}_{c_{0}}=\boldsymbol{W}_{\text {vert }} \neq \mathbf{0}$. In this case the solution $\boldsymbol{U}=0$ is a quasi-equilibrium.

It is convenient to rewrite equations (11)-(16) as evolution equations for two-dimensional perturbations about this equilibrium state. We denote the perturbations by $\left(\boldsymbol{U}^{\prime}, P^{\prime}, T^{\prime}, C^{\prime}\right)$ and introduce the following streamfunction representations:

$$
\begin{aligned}
& U_{x}^{\prime}=-\partial \psi^{\prime} / \partial z, \quad U_{z}^{\prime}=\partial \psi^{\prime} / \partial x, \\
& W_{T_{x}}^{\prime}=-\partial \psi_{1}^{\prime} / \partial z, \quad W_{T_{z}}^{\prime}=\partial \psi_{1}^{\prime} / \partial x, \\
& W_{C_{x}}^{\prime}=-\partial \psi_{2}^{\prime} / \partial z, \quad W_{C_{z}}^{\prime}=\partial \psi_{2}^{\prime} / \partial x .
\end{aligned}
$$

As a result a positive streamfunction corresponds to a clockwise cell. Eliminating $p^{\prime}$ we obtain

$$
\begin{aligned}
& \frac{\partial}{\partial t}\left(\begin{array}{c}
\Delta \psi^{\prime} \\
T^{\prime} \\
C^{\prime}
\end{array}\right)=\left(\begin{array}{ccc}
\Delta^{2} & \mathrm{Gr} \frac{\partial}{\partial x} & -\mathrm{Gr} \frac{\partial}{\partial x} \\
-\frac{\partial}{\partial z} & \frac{\Delta}{\mathrm{Pr}} & 0 \\
-\frac{\partial}{\partial z} & 0 & \frac{\Delta}{\mathrm{Sc}}
\end{array}\right)\left(\begin{array}{c}
\prime \\
T^{\prime} \\
C^{\prime}
\end{array}\right)+\left(\begin{array}{l}
N_{1}\left(\psi^{\prime}, \psi^{\prime}\right) \\
N_{2}\left(\psi^{\prime}, T^{\prime}\right) \\
N_{2}\left(\psi^{\prime}, C^{\prime}\right)
\end{array}\right) \\
&-R_{\mathrm{v}}\left(\begin{array}{c}
N_{3}\left(\psi_{1}^{\prime}-{ }_{2}^{\prime}, T^{\prime}-C^{\prime}\right)-N_{1}\left(\psi_{1}^{\prime}-{ }_{2}, \psi_{1}^{\prime}-{ }_{2}^{\prime}\right) \\
0 \\
0
\end{array}\right) \\
& \Delta \psi_{1}^{\prime}=\sin \alpha \frac{\partial T^{\prime}}{\partial x}-\cos \alpha \frac{\partial T^{\prime}}{\partial z}, \\
& \Delta \psi_{2}^{\prime}=\sin \alpha \frac{\partial C^{\prime}}{\partial x}-\cos \alpha \frac{\partial C^{\prime}}{\partial z},
\end{aligned}
$$


where for all pairs $(f, g)$ of real functions

$$
\begin{aligned}
N_{1}(f, f)= & \frac{\partial f}{\partial z}\left(\frac{\partial^{3} f}{\partial x \partial z^{2}}+\frac{\partial^{3} f}{\partial x^{3}}\right)-\frac{\partial f}{\partial x}\left(\frac{\partial^{3} f}{\partial z \partial x^{2}}+\frac{\partial^{3} f}{\partial z^{3}}\right), \\
N_{2}(f, g)= & \frac{\partial f}{\partial z} \frac{\partial g}{\partial x}-\frac{\partial f}{\partial x} \frac{\partial g}{\partial z} \\
N_{3}(f, g)= & -\sin \alpha\left(\frac{\partial^{2} f}{\partial x^{2}} \frac{\partial g}{\partial z}+\frac{\partial f}{\partial x} \frac{\partial^{2} g}{\partial x \partial z}-\frac{\partial^{2} f}{\partial x \partial z} \frac{\partial g}{\partial x}-\frac{\partial f}{\partial z} \frac{\partial^{2} g}{\partial x^{2}}\right) \\
& +\cos \alpha\left(\frac{\partial^{2} g}{\partial z^{2}} \frac{\partial f}{\partial x}+\frac{\partial g}{\partial z} \frac{\partial^{2} f}{\partial x \partial z}-\frac{\partial^{2} g}{\partial x \partial z} \frac{\partial f}{\partial z}-\frac{\partial g}{\partial x} \frac{\partial^{2} f}{\partial z^{2}}\right) .
\end{aligned}
$$

At the boundaries of the cavity the streamfunctions vanish and thus

$$
\begin{aligned}
& \left(\frac{\partial \psi^{\prime}}{\partial x}\right)_{x=0,1}=\left(\frac{\partial \psi^{\prime}}{\partial z}\right)_{z=0, A}=\left(\psi^{\prime}\right)_{\partial \Omega}=0 . \\
& T^{\prime}(x=1, z)=C^{\prime}(x=1, z)=T^{\prime}(x=0, z)=C^{\prime}(x=0, z)=0 \quad \forall z \in[0, A], \\
& \frac{\partial T^{\prime}}{\partial z}(x, z=0, A)=\frac{\partial C^{\prime}}{\partial z}(x, z=0, A)=0 \quad \forall x \in[0,1], \\
& \quad{ }_{1}^{\prime}={ }_{2}^{\prime}=0 \quad \text { along } \partial \Omega .
\end{aligned}
$$

Eqs. (27) $-(29)$ with boundary conditions (33)-(36) are invariant under rotations by $\pi$ about the point $\left(\frac{1}{2}, A / 2\right)$. This rotation is described by the operator $S$ defined by

$$
S\left(\begin{array}{r}
\prime \\
T^{\prime} \\
C^{\prime} \\
1 \\
1 \\
1 \\
2
\end{array}\right)(x, z)=\left(\begin{array}{r}
\prime \\
-T^{\prime} \\
-C^{\prime} \\
1 \\
1 \\
2 \\
2
\end{array}\right)(1-x, A-z)
$$

and is a generalized reflection since $S^{2}=1$. The resulting symmetry group $Z_{2} \equiv\{I, S\}$ plays an important role in the bifurcation analysis described below. In particular, it is known that in the presence of this symmetry group (Crawford and Knobloch, 1991), the conduction state can only lose stability to states that are either symmetric or antisymmetric with respect to $S$. The former occurs when the marginally stable eigenfunction is invariant under $S$, the latter when it breaks invariance under $S$.

\subsection{Analytical results}

In this section we summarize a number of results that can be obtained analytically. These results are based on a severe truncation of a Galerkin expansion but nonetheless describe correctly the qualitative behavior of the solutions as a function of the system parameters. Precise and complete bifurcation diagrams for specific parameter choices are presented in Section 5. 
The linearized time-dependent equations are obtained by neglecting the nonlinear terms. The resulting linear problem is independent of the vibrational Grashof number (i.e., $T_{0}=C_{0}$ and $\boldsymbol{W}_{T_{0}}=\boldsymbol{W}_{c_{0}}$ ), and the computation of the vibrational streamfunctions decouples. Solving the corresponding equations (27) gives $T^{\prime}$ and $C^{\prime}$. The vibrational part of the velocity represented by $\psi_{1}^{\prime}$ and $\psi_{2}^{\prime}$ is then obtained from Eqs. (28) and (29). Because of the physical boundary conditions, the value of the thermal Grashof number Gr is positive. Ghorayeb and Mojtabi (1997) showed that in the static case the onset of convection occurs at a critical Grashof number $\mathrm{Gr}^{(0)}$ given by $\mathrm{Gr}^{(0)}|\mathrm{Sc}-\mathrm{Pr}|=17172$ (for $A=1$ ) and 7650 (for $A=2.6$ ). This result continues to hold in the presence of vibration. In fact the linear stability results for the static case (without vibration) obtained by Ghorayeb and Mojtabi (1997) and Bardan et al. (2000) are recovered for arbitrary values of $A$. These authors show that the primary bifurcations are always stationary.

The linear stability problem is solved by means of a Galerkin method using the following expansions:

$$
\begin{aligned}
& { }^{\prime}(x, z)=\sum_{i=0}^{n} \sum_{j=0}^{m} a_{i j} \sin (\pi x) \sin (i \pi x) \sin (\pi z / A) \sin (j \pi z / A), \\
& T^{\prime}(x, z)=\sum_{i=0}^{n} \sum_{j=0}^{m} b_{i j} \sin (i \pi x) \cos (j \pi z / A), \\
& C^{\prime}(x, z)=\sum_{i=0}^{n} \sum_{j=0}^{m} c_{i j} \sin (i \pi x) \cos (j \pi z / A), \\
& { }_{1}^{\prime}(x, z)=\sum_{i=0}^{n} \sum_{j=0}^{m} d_{i j} \sin (i \pi x) \sin (j \pi z / A), \\
& { }_{2}^{\prime}(x, z)=\sum_{i=0}^{n} \sum_{j=0}^{m} e_{i j} \sin (i \pi x) \sin (j \pi z / A) .
\end{aligned}
$$

Due to the $\boldsymbol{Z}_{2}$ symmetry the associated eigenmodes are either symmetric with respect to $S$, i.e., $S\left(\psi^{\prime}, T^{\prime}, C^{\prime}, \psi_{1}^{\prime}, \psi_{2}^{\prime}\right)=\left(\psi^{\prime}, T^{\prime}, C^{\prime}, \psi_{1}^{\prime}, \psi_{2}^{\prime}\right)$, or antisymmetric, i.e., $S\left(\psi^{\prime}, T^{\prime}, C^{\prime}, \psi_{1}^{\prime}, \psi_{2}^{\prime}\right)=-\left(\psi^{\prime}, T^{\prime}, C^{\prime}, \psi_{1}^{\prime}, \psi_{2}^{\prime}\right)$. The symmetric eigenmodes contain an odd number of cells whereas the antisymmetric ones contain an even number of cells. Eqs. (38)-(42) indicate that the contribution to the symmetric modes comes from terms with $i+j$ even while the contribution to the antisymmetric modes comes from terms with $i+j$ odd. We used the software MAPLE to find the values of $\mathrm{Gr}_{c}, a_{i j}, b_{i j}, c_{i j}, d_{i j}$ and $e_{i j}$ at which a bifurcation to a symmetric mode $(A=1)$ or an antisymmetric one $(A=2.6)$ occurs. For the symbolic computations described below we use $n=m=4$ for $\psi^{\prime}, n=m=3$ for $T^{\prime}$ and $C^{\prime}$ and $n=m=2$ for $\psi_{1}^{\prime}$ and $\psi_{2}^{\prime}$.

In the next section we study first the codimension one cases arising when $\mathrm{Gr}$ is increased for $A=1$ and 2.6. We use center manifold reduction (Guckenheimer and Holmes, 1983) to describe the weakly nonlinear problem with particular attention devoted to its dependence on the vibrational Grashof number $R_{\mathrm{v}}$ and the vibration angle $\alpha$. We only consider the case $\mathrm{Sc}>\operatorname{Pr}$ corresponding to most binary mixtures, i.e., Le $>1$. 


\section{Nonlinear analysis}

\subsection{Weakly nonlinear analysis}

In this section we use a multiple scale analysis to reduce Eqs. (27)-(29) to a simpler form, called a normal form, valid near onset of the primary instability, i.e., for $\left|\mathrm{Gr}-\mathrm{Gr}^{(0)}\right| / \mathrm{Gr}^{(0)} \ll 1$. We expand the perturbations in powers of a small parameter $\varepsilon>0$,

$$
\psi^{\prime}=\varepsilon \psi^{(1)}+\varepsilon^{2} \psi^{(2)}+\cdots,
$$

with similar expressions for $T^{\prime}, C^{\prime}, \psi_{1}^{\prime}, \psi_{2}^{\prime}$, and write

$$
\begin{aligned}
& \frac{\partial}{\partial t}=\varepsilon \frac{\partial}{\partial t^{(1)}}+\varepsilon^{2} \frac{\partial}{\partial t^{(2)}}+\cdots, \\
& \mathrm{Gr}=\mathrm{Gr}^{(0)}+\varepsilon \mathrm{Gr}^{(1)}+\varepsilon^{2} \mathrm{Gr}^{(2)}+\cdots .
\end{aligned}
$$

At order $\varepsilon$ we recover the linear eigenvalue problem. Since $C^{(1)}=\operatorname{Le} T^{(1)}$, the critical eigenmode can be written in the form

$$
\left(\begin{array}{c}
(1) \\
T^{(1)} \\
C^{(1)} \\
(1) \\
1 \\
(1)
\end{array}\right)=K\left(t^{(1)}\right)\left(\begin{array}{c}
f_{1}(x, z) \\
\operatorname{Pr} f_{2}(x, z) \\
\operatorname{Sc} f_{2}(x, z) \\
\operatorname{Pr} f_{3}(x, z) \\
\operatorname{Sc} f_{3}(x, z)
\end{array}\right),
$$

where the amplitude $K$ depends on the slow time $t^{(1)}$ and the functions $f_{i}$ depend only on the spatial variables. These functions and the corresponding critical Grashof number $\mathrm{Gr}^{(0)}$ are computed as described in Section 3.

At order $\varepsilon^{2}$ we obtain

$$
\begin{aligned}
& \left(\begin{array}{cccc}
\Delta^{2} & \mathrm{Gr}^{(0)} \frac{\partial}{\partial x} & -\mathrm{Gr}^{(0)} \frac{\partial}{\partial x} \\
-\frac{\partial}{\partial z} & \frac{\Delta}{\operatorname{Pr}} & 0 & \\
-\frac{\partial}{\partial z} & 0 & \frac{\Delta}{\mathrm{Sc}}
\end{array}\right)\left(\begin{array}{l}
\psi^{(2)} \\
T^{(2)} \\
C^{(2)}
\end{array}\right) \\
& =-\left(\begin{array}{lll}
0 & \mathrm{Gr}^{(1)} \frac{\partial}{\partial x} & -\mathrm{Gr}^{(1)} \frac{\partial}{\partial x} \\
0 & 0 & 0 \\
0 & 0 & 0
\end{array}\right)\left(\begin{array}{l}
\psi^{(1)} \\
T^{(1)} \\
C^{(1)}
\end{array}\right)-\left(\begin{array}{l}
N_{1}\left(\psi^{(1)}, \psi^{(1)}\right) \\
N_{2}\left(\psi^{(1)}, T^{(1)}\right) \\
N_{2}\left(\psi^{(1)}, C^{(1)}\right)
\end{array}\right)+\frac{\partial}{\partial t^{(1)}}\left(\begin{array}{c}
\Delta \psi^{(1)} \\
T^{(1)} \\
C^{(1)}
\end{array}\right) \\
& -R_{\mathrm{v}}\left(\begin{array}{c}
N_{1}\left(\psi_{1}^{(1)}-{ }_{2}^{(1)}, \psi_{1}^{(1)}-{ }_{2}^{(1)}\right)-N_{3}\left(\psi_{1}^{(1)}-\psi_{2}^{(1)}, T^{(1)}-C^{(1)}\right) \\
0 \\
0
\end{array}\right) .
\end{aligned}
$$

The solvability condition for these equations now yields the evolution equation. To obtain this equation we need the solution to the adjoint eigenvalue problem

$$
\left(\begin{array}{ccc}
\Delta^{2} & \frac{\partial}{\partial z} & \frac{\partial}{\partial z} \\
-\mathrm{Gr}^{*(0)} \frac{\partial}{\partial x} & \frac{\Delta}{\operatorname{Pr}} & 0 \\
\operatorname{Gr}^{*(0)} \frac{\partial}{\partial x} & 0 & \frac{\Delta}{\mathrm{Sc}}
\end{array}\right)\left(\begin{array}{c}
* \\
T^{*} \\
C^{*}
\end{array}\right)=\left(\begin{array}{l}
0 \\
0 \\
0
\end{array}\right)
$$


with identical boundary conditions. Thus $\mathrm{Gr}^{*(0)}=\mathrm{Gr}^{(0)}$ and

$$
\left(\begin{array}{c}
{ }^{*} \\
T^{*} \\
C^{*}
\end{array}\right)=\left(\begin{array}{c}
(\operatorname{Pr}-\mathrm{Sc}) f_{1}^{*}(x, z) \\
\operatorname{Pr} f_{2}^{*}(x, z) \\
-\operatorname{Sc} f_{2}^{*}(x, z)
\end{array}\right) .
$$

The solvability condition is therefore

$$
(e+(\mathrm{Pr}+\mathrm{Sc}) f) \frac{\partial K}{\partial t^{(1)}}=\mathrm{Gr}^{(1)}(\mathrm{Pr}-\mathrm{Sc}) a K+\left(b+R_{\mathrm{v}}(\mathrm{Sc}-\mathrm{Pr})^{2} c+(\mathrm{Pr}+\mathrm{Sc}) d\right) K^{2}
$$

with $\mathrm{Gr}^{(1)}$ indicating the distance from onset. The coefficients are given by the expressions

$$
\begin{aligned}
& a=\iint_{\Omega} \frac{\partial f_{2}}{\partial x} f_{1}^{*} \mathrm{~d} x \mathrm{~d} z, \\
& b=\iint_{\Omega} N_{1}\left(f_{1}, f_{1}\right) f_{1}^{*} \mathrm{~d} x \mathrm{~d} z, \\
& c=\iint_{\Omega}\left(N_{1}\left(f_{3}, f_{3}\right)-N_{3}\left(f_{3}, f_{2}\right)\right) f_{1}^{*} \mathrm{~d} x \mathrm{~d} z, \\
& d=\iint_{\Omega} N_{2}\left(f_{1}, f_{2}\right) f_{2}^{*} \mathrm{~d} x \mathrm{~d} z, \\
& e=\iint_{\Omega} \Delta f_{1} f_{1}^{*} \mathrm{~d} x \mathrm{~d} z, \\
& f=\iint_{\Omega} f_{2} f_{2}^{*} \mathrm{~d} x \mathrm{~d} z,
\end{aligned}
$$

where the asterisk denotes the adjoint eigenfunctions. In the following two subsections we summarize the results obtained analytically from the truncated Galerkin equations.

\subsection{Case $A=1$ : The symmetric case $S\left(f_{1}(x, z)\right)=f_{1}(x, z)$.}

For aspect ratio $A=1$ the critical eigenmode is invariant under the symmetry $S$. The bifurcation is therefore expected to be transcritical. Our symbolic computations confirm this expectation, giving $a=-0.142, b=-159.3, c=c(\alpha), d=184960, e=41.5$ and $f=126.7$. Consequently, the purely diffusive solution $(K=0)$ is stable if and only if $\mathrm{Gr}^{(1)}<0$, i.e., for Grashof numbers smaller than the critical thermal Grashof number, while the convective solution $(K \neq 0)$ is stable for $\mathrm{Gr}^{(1)}>0$ and unstable for $\mathrm{Gr}^{(1)}<0$. Fig. 2 shows the coefficient $c$ as a function of the angle of vibration $\alpha$. Note that this function is $\pi$-periodic because vibration at angle $\alpha$ or $\alpha+\pi$ is the same. The convective solution is given by

$$
(x, z) \approx \varepsilon \psi^{(1)}=f_{1}(x, z) \frac{a}{b+R_{\mathrm{v}}(\mathrm{Sc}-\mathrm{Pr})^{2} c+(\mathrm{Pr}+\mathrm{Sc}) d}\left(\mathrm{Gr}-\mathrm{Gr}^{(0)}\right)(\mathrm{Sc}-\mathrm{Pr}) .
$$

In the following we refer to the branch that bifurcates supercritically (subcritically) from the quasiequilibrium state as the supercritical (subcritical) branch, regardless of its subsequent appearance. 


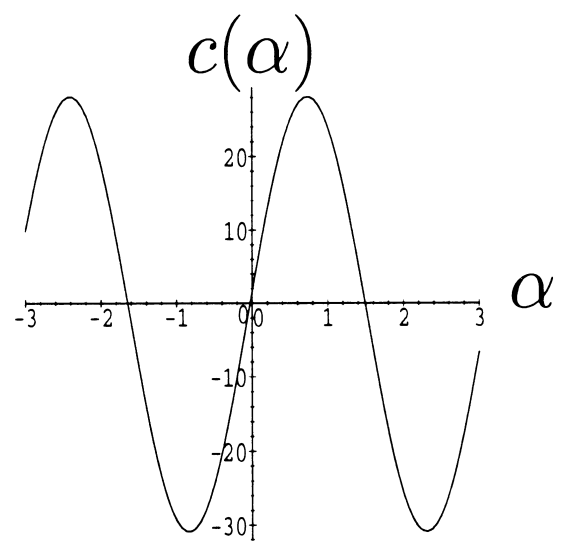

Fig. 2. The function $c(\alpha)$ obtained using MAPLE with $n=m=4$ for $\psi$ and $n=m=3$ for $T$ and $C$.

The supercritical branch is initially stable, while the subcritical one is initially unstable. Subsequent saddle-node and/or Hopf bifurcations may change these stability properties.

For $\operatorname{Pr}=1, \mathrm{Sc}=11$ and $R_{\mathrm{v}}=0$ we find that the convective branch has a very small slope in the $\left(\psi\left(\frac{1}{2}, \frac{1}{2}\right), \mathrm{Gr}\right)$ plane, of the order of $10^{-6}$. This result can be confirmed by direct numerical simulation. Since the numerical solution of the linear problem shows that $f_{1}>0$ at $x=z=\frac{1}{2}$ whenever Le $>1$, while the solution of the adjoint problem implies that $a<0, d>0$ and $b<0$ with $\operatorname{Pr}+\operatorname{Sc}>-b / d(\approx$ $\left.10^{-3}\right)$, it follows that on the stable supercritical branch $\psi\left(\frac{1}{2}, \frac{1}{2}\right)<0$ and hence that the corresponding flow is counterclockwise. This is because when Le $>1$ heat diffuses faster than concentration and consequently the direction of motion is determined by the concentration gradients. At the left of the cavity these carry heavier fluid from the top to the bottom producing a counterclockwise cell. As the strength of the vibration increases this picture gradually changes because of the $\alpha$ dependence of $c$. If $\alpha=0$ or $1.4(\bmod \pi)$ the coefficient $c$ vanishes and the resulting bifurcation diagram is independent of the vibration strength.

If $0<\alpha<1.4(\bmod \pi) c>0$ and the slope decreases (in magnitude) as $R_{\mathrm{v}}$ increases. Finally, if $1.4<\alpha<\pi(\bmod \pi) c<0$ and the magnitude of the slope instead increases with $R_{\mathrm{v}}$ and the slope becomes vertical at

$$
R_{\mathrm{v}}=-\frac{b+(\mathrm{Pr}+\mathrm{Sc}) d}{(\mathrm{Sc}-\mathrm{Pr})^{2} c}>0 .
$$

At this point the perturbation expansion breaks down and a different scaling must be employed. This scaling brings in a cubic term in the amplitude $K$, indicating that at degeneracy the bifurcation is a pitchfork. The unfolding of this degeneracy is correctly described by including a small quadratic term in $K$ as in Eq. (50). Golubitsky and Schaeffer (1985) discuss additional phenomena described by the universal unfolding of the pitchfork. In the present problem these require relaxing the requirement $N=-1$.

Although for $A=1$ the mode truncation used predicts that $\mathrm{Gr}^{(0)}(\mathrm{Sc}-\mathrm{Pr})=19040$ (compared with the exact value $\left.\mathrm{Gr}^{(0)}(\mathrm{Sc}-\mathrm{Pr})=17172\right)$ the analytical calculation captures correctly the qualitative dependence of the solutions on the system parameters. Numerically accurate bifurcation diagrams for vertical vibration and $\mathrm{Le}=11,51$ can be found in Section 5.2.2. 
4.3. Case $A=2.6$ : The antisymmetric case $S\left(f_{1}(x, z)\right)=-f_{1}(x, z)$.

When $A=2.6$ the critical eigenmode is antisymmetric

$$
S\left(\psi^{\prime}\left(x, z, t^{(1)}\right)\right) \equiv \psi^{\prime}\left(1-x, A-z, t^{(1)}\right)=-\psi^{\prime}\left(x, z, t^{(1)}\right)
$$

and we now expect a nondegenerate pitchfork bifurcation. Our symbolic calculations confirm that $b=c=d=0$ for all values of $m$ and $n$. Thus $\mathrm{Gr}^{(1)}=0(K \neq 0)$. Consequently, $K$ does not depend on $t^{(1)}$.

In order to obtain the first nonzero $\operatorname{Gr}^{(i)}$ for $i=\{1,2,3, \ldots, n\}$, we solve the order $\varepsilon^{2}$ problem for (2), $T^{(2)}$ and $C^{(2)}$. The solution takes the form

$$
\left(\begin{array}{c}
(2) \\
T^{(2)} \\
C^{(2)} \\
(2) \\
1(2) \\
2
\end{array}\right)=K^{2}\left(t^{(2)}\right)\left(\begin{array}{l}
g_{1}\left(x, z, \operatorname{Pr}, \mathrm{Sc}, \alpha, R_{\mathrm{v}}\right) \\
g_{2}\left(x, z, \operatorname{Pr}, \mathrm{Sc}, \alpha, R_{\mathrm{v}}\right) \\
g_{3}\left(x, z, \operatorname{Pr}, \mathrm{Sc}, \alpha, R_{\mathrm{v}}\right) \\
g_{4}\left(x, z, \operatorname{Pr}, \mathrm{Sc}, \alpha, R_{\mathrm{v}}\right) \\
g_{5}\left(x, z, \operatorname{Pr}, \mathrm{Sc}, \alpha, R_{\mathrm{v}}\right)
\end{array}\right) .
$$

At $\operatorname{order} \varepsilon^{3}$, we obtain

$$
\begin{aligned}
\left(\begin{array}{ccc}
\Delta^{2} & \mathrm{Gr}^{(0)} \frac{\partial}{\partial x} & -\mathrm{Gr}^{(0)} \frac{\partial}{\partial x} \\
-\frac{\partial}{\partial z} & \frac{\Delta}{\operatorname{Pr}} & 0 \\
-\frac{\partial}{\partial z} & 0 & \frac{\Delta}{\mathrm{Sc}}
\end{array}\right)\left(\begin{array}{l}
\psi^{(3)} \\
T^{(3)} \\
C^{(3)}
\end{array}\right) \\
=-\left(\begin{array}{lll}
0 & \mathrm{Gr}^{(2)} \frac{\partial}{\partial x} & -\mathrm{Gr}^{(2)} \frac{\partial}{\partial x} \\
0 & 0 & 0 \\
0 & 0 & 0
\end{array}\right)\left(\begin{array}{l}
\psi^{(2)} \\
T^{(2)} \\
C^{(2)}
\end{array}\right)-\left(\begin{array}{l}
N_{1}\left(\psi^{(1)}, \psi^{(2)}\right) \\
N_{2}\left(\psi^{(1)}, T^{(2)}\right) \\
N_{2}\left(\psi^{(1)}, C^{(2)}\right)
\end{array}\right)-\left(\begin{array}{l}
N_{1}\left(\psi^{(2)}, \psi^{(1)}\right) \\
N_{2}\left(\psi^{(2)}, T^{(1)}\right) \\
N_{2}\left(\psi^{(2)}, C^{(1)}\right)
\end{array}\right) \\
-R_{\mathrm{v}}\left(\begin{array}{cc}
N_{1}\left(\psi_{1}^{(1)}-\psi_{2}^{(1)}, \psi_{1}^{(2)}-{ }_{2}^{(2)}\right)-N_{3}\left(\psi_{1}^{(1)}-\psi_{2}^{(1)}, T^{(2)}-C^{(2)}\right) \\
0 \\
0
\end{array}\right)+\frac{\partial}{\partial t^{(2)}}\left(\begin{array}{c}
\Delta \psi^{(1)} \\
T^{(1)} \\
C^{(1)}
\end{array}\right) .
\end{aligned}
$$

The solvability condition now yields

$$
\left(e_{p}+(\operatorname{Pr}+\mathrm{Sc}) f_{p}\right) \frac{\partial K}{\partial t^{(2)}}=a_{p} \mathrm{Gr}^{(2)}(\mathrm{Sc}-\mathrm{Pr}) K-g_{p}\left(\operatorname{Pr}, \mathrm{Sc}, \alpha, R_{\mathrm{v}}\right) K^{3}
$$

where $a_{p} \approx 7.41, e_{p} \approx 2380, f_{p} \approx 8845$ and $g_{p} \equiv g_{p}\left(\operatorname{Pr}, \mathrm{Sc}, \alpha, R_{\mathrm{v}}\right)$.

In the static case $\left(R_{\mathrm{V}}=0\right)$ the pitchfork bifurcation is subcritical $\left(g_{p}<0\right)$ for any value of $\operatorname{Pr}$ and $\mathrm{Sc}$, and the emerging solutions are unstable. However, as $\alpha$ and $R_{\mathrm{v}}$ vary the pitchfork can be transformed into a supercritical one and the convective solutions stabilized at small amplitude. This 

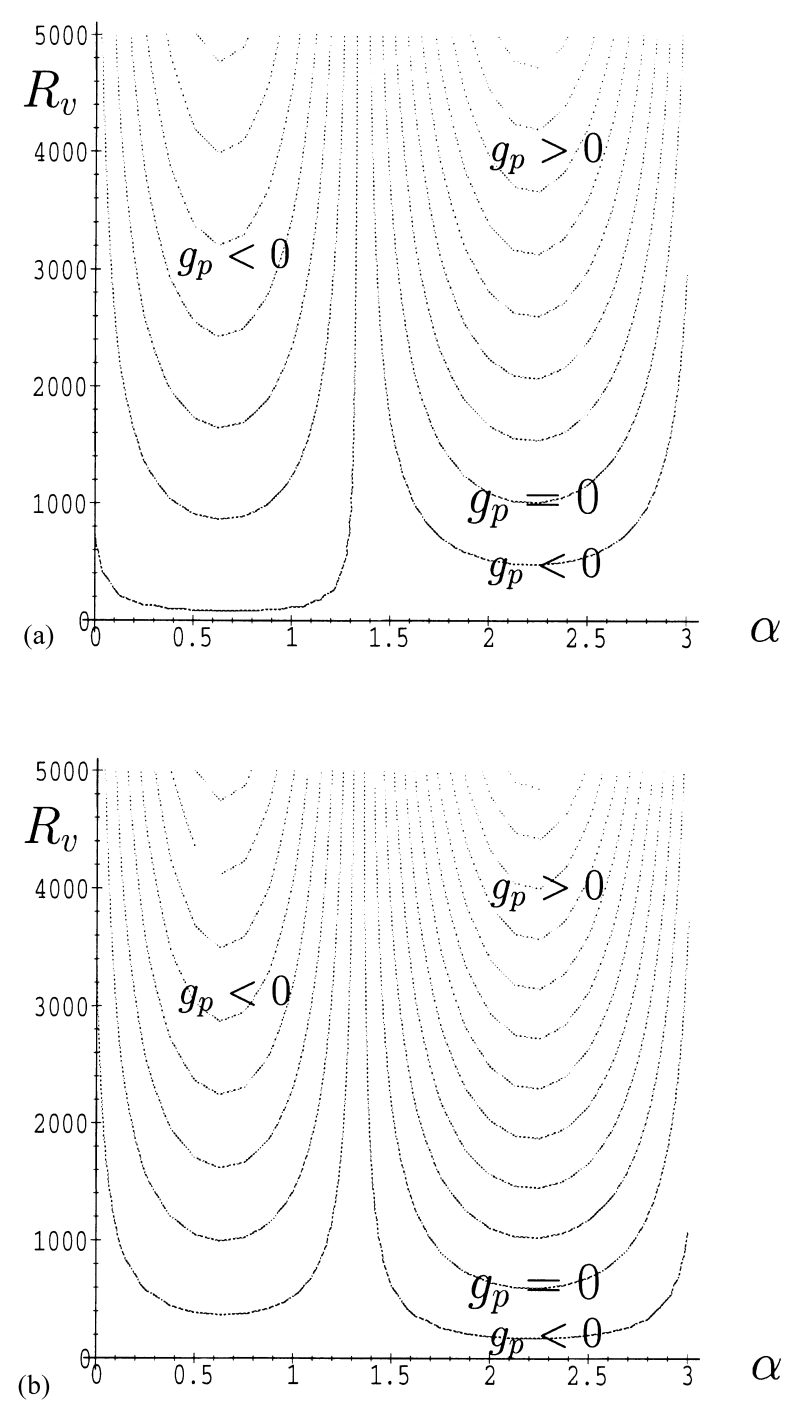

Fig. 3. Contour plot of $g_{p}\left(\operatorname{Pr}, \mathrm{Sc}, \alpha, R_{\mathrm{v}}\right)$ obtained using MAPLE with $n=m=4$ for $\psi$ and $n=m=3$ for $T$ and $C$ for $\operatorname{Pr}=1$ and (a) $\mathrm{Sc}=11$, (b) $\mathrm{Sc}=51$. For $1.4<\alpha<3.1, g_{p}$ is initially negative but becomes positive as $R_{\mathrm{v}}$ increases.

solution is given by

$$
2 \approx \varepsilon^{2} \psi^{(1)^{2}}=\frac{a_{p} f_{1}^{2}}{g_{p}\left(\mathrm{Pr}, \mathrm{Sc}, \alpha, R_{\mathrm{v}}\right)}\left(\mathrm{Gr}-\mathrm{Gr}^{(0)}\right)(\mathrm{Sc}-\mathrm{Pr}) .
$$

Fig. 3 shows a contour plot of $g_{p}$ as a function of both $\alpha$ and $R_{\mathrm{v}}$ when $\operatorname{Pr}=1$ and (a) Sc $=11$, (b) $\mathrm{Sc}=51$, again obtained from the truncated Galerkin expansion. This function describes correctly the functional dependence of the solution on the system parameters. 


\section{Numerical results}

\subsection{Numerical method}

The system of equations (11)-(16) was solved numerically using a spectral method (Canuto et al., 1987). The method used is based on the projection diffusion algorithm developed for solving the 2D-3D unsteady incompressible Navier-Stokes equations (Khallouf, 1995). Temporal integration consists of a semi-implicit second-order finite difference approximation. The linear (viscous) terms are treated implicitly using a second-order backward Euler scheme, while a second-order explicit AdamsBashforth scheme is employed for the nonlinear (advective) parts. When applied to an advectiondiffusion equation such as

$$
\frac{\partial f}{\partial t}+\boldsymbol{U} \cdot \nabla f=\alpha \Delta f
$$

the method leads to

$$
\frac{\frac{3}{2} f^{n+1}-2 f^{n}+\frac{1}{2} f^{n-1}}{\delta t}=\alpha \Delta f^{n+1}-2(\boldsymbol{U} \cdot \nabla f)^{n}-(\boldsymbol{U} \cdot \nabla f)^{n-1} .
$$

This equation can be written in the form of the Helmholtz equation

$$
(\Delta-h) f^{n+1}=s,
$$

where $h=3 / 2 \alpha \delta t$ is the Helmholtz constant and $s$ is a scalar quantity containing all the terms known at time $t_{n}=n \delta t$ ( $n$ is the time level and $\delta t$ is the time step). The temporal integration therefore transforms the system into a Helmholtz problem arising from the advection-diffusion equations (13) and (14) coupled to the Poisson problems (15) and (16) with appropriate boundary conditions. The latter are solved using the Uzawa (or Poisson-like) formulation of Azaiez et al. (1994). The NavierStokes equations (11)-(12) are transformed into a generalized Stokes problem and solved by the projection-diffusion method of Khallouf (1995). All the subproblems obtained are either Helmholtz or Poisson-like operators. A high-accuracy spectral method, namely one utilizing Legendre collocation points, is used in the spatial discretization of the Helmholtz and Poisson-like operators. Successive diagonalization is implemented to invert these operators. We mention that the Stokes and DarcyEuler solvers are direct and guarantee an accurate spectral solution with divergence-free solenoidal fields over the entire domain, including the boundaries. For the calculations discussed in this paper a grid with $15 \times 15$ mesh points in the $(x, z)$ domain suffices to describe accurately the flow for $\mathrm{Le}=11$. We selected a $25 \times 25$ grid for our calculations at $\mathrm{Le}=11$ and $35 \times 35$ at $\mathrm{Le}=51$.

\subsection{Numerical results}

For the numerical study, we chose $\mathrm{Sc}=11$ and $\mathrm{Pr}=1$ in order to investigate a case in which there is a substantial difference between the thermal and solutal disturbances. Some results for Le $=51$ are also described. We consider two particular geometries, a square cavity $(A=1)$ and a rectangular cavity with $A=2.6$. We begin with zero-gravity results, i.e., with thermo-vibrational convection, followed by a discussion of the interaction between vibrational and static gravity driven convection. 


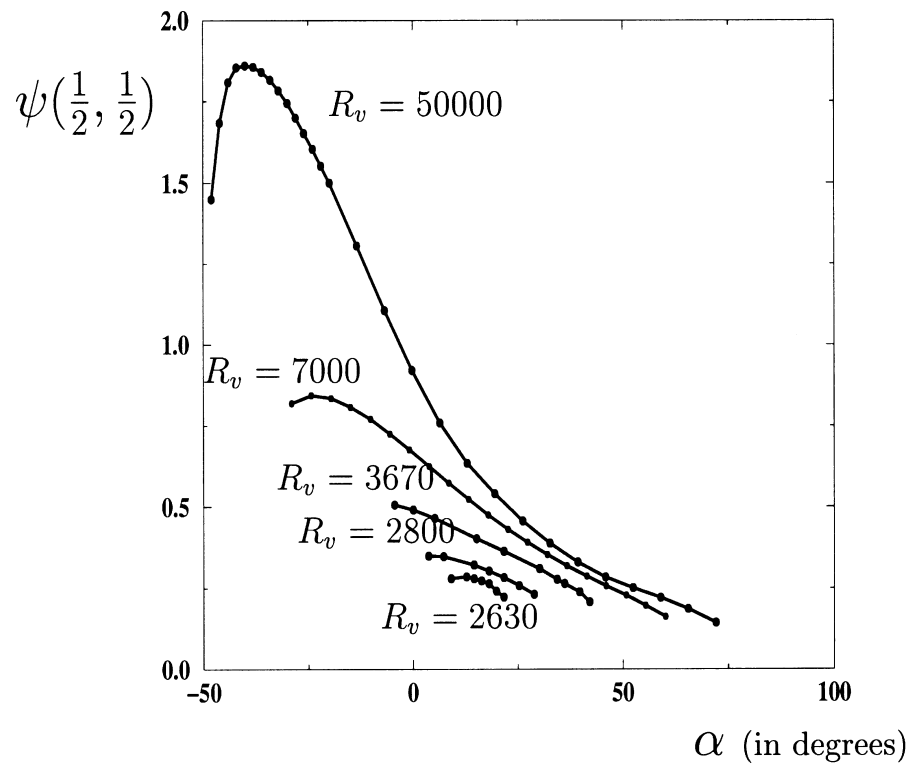

Fig. 4. Graph of $\psi\left(\frac{1}{2}, \frac{1}{2}\right)$ versus $\alpha$ (degrees) for finite amplitude solutions in zero gravity $(\mathrm{Gr}=0)$ and different values of $R_{\mathrm{v}}$ when $\mathrm{Le}=11, \mathrm{Pr}=1$. The solid points denote the calculated values. Resolution is $25 \times 25$.

\subsubsection{Zero gravity results for $A=1$}

For the square cavity the theoretical critical Grashof number is given by $\mathrm{Gr}^{(0)}(\mathrm{Sc}-\operatorname{Pr})=17172$, i.e., $\mathrm{Gr}^{(0)}=1717$. The corresponding instability is a steady one (see Sections 2 and 3). When $\mathrm{Gr}=0$ the system is controlled by the vibration angle $\alpha$ and the vibration amplitude $R_{\mathrm{v}}$. Computations were done for $0<R_{\mathrm{v}}<50000$ for $\alpha$ between $\frac{\pi}{2}$ to $-\frac{\pi}{2}$.

The first set of computations was performed with initial data for the diffusive solution and confirmed the stability of the equilibrium or quasi-equilibrium solutions predicted by linear theory. For Le $>1$ larger amplitude solutions evolved either to this state or to thermo-vibrational convection consisting of a clockwise one-cell flow, depending on $\alpha$ and $R_{\mathrm{v}}$. The results are summarized in Fig. 4 for selected values of $R_{\mathrm{v}}\left(0<R_{\mathrm{v}}<50000\right)$. The figure shows that the range of angles for which a convective solution exists diminishes with decreasing $R_{\mathrm{v}}$ and vanishes at $R_{\mathrm{vc}}=2610$, obtained for $\alpha=16^{\circ}$. Note that, at high values of $R_{\mathrm{v}}$, the flow amplitude passes through a maximum for negative angles. Additional numerical simulations showed that for $\alpha>\alpha_{\mathrm{c}}=73^{\circ}$ convective motion is suppressed regardless of the value of $R_{\mathrm{v}}$. For comparison, for a horizontally vibrating cavity the critical vibrational Rayleigh number is $R_{\mathrm{v}}=2860$ whereas the conductive solution is unconditionally stable when vibrated vertically. With two-dimensional steady flow, the streamlines correspond to lines of constant streamfunction and a single contour map gives a complete portrayal of the flow field. Fig. 5 shows that the flow consists of a clockwise rotating single cell. In all three fields (velocity, temperature and concentration) the resulting thermo-vibrational flow resembles natural convection.

\subsubsection{Interaction between vibration and gravity driven convection for $A=1$}

In view of the weakly nonlinear results of Section 4 we now turn to three representative cases $\left(\alpha=0, \alpha=\frac{\pi}{4}\right.$ and $\left.\alpha=\frac{\pi}{2}\right)$ illustrating the different types of interaction between vibration and static 

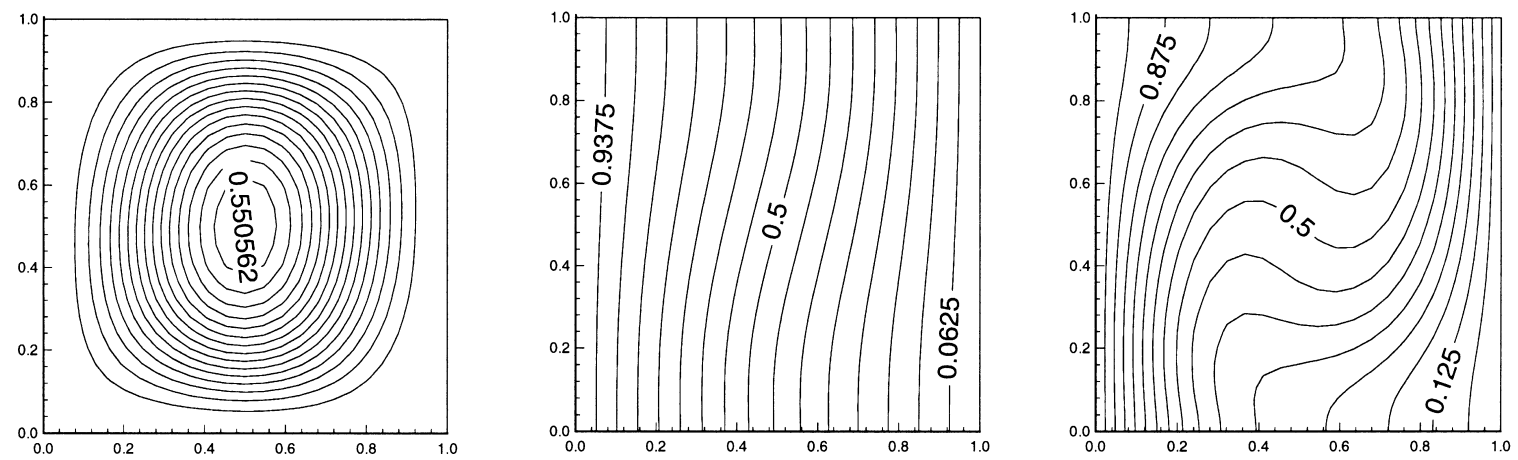

Fig. 5. Streamlines, isotherms and isoconcentration lines in zero gravity for $R_{\mathrm{v}}=5000$ and horizontal vibration (Le $=11$, $\operatorname{Pr}=1, A=1)$. Resolution is $25 \times 25$.

gravity driven convection. We are particularly interested in the location of the turning point on the subcritical convective branch.

Horizontal vibration $(\alpha=0)$ : When the vibration is along the static gradient (Fig. 6) the supercritical regime branches from the pure diffusive state at $\operatorname{Gr}(\mathrm{Sc}-\mathrm{Pr})=17161$ (for comparison the value obtained by linear stability analysis is 17172). The numerical calculation confirms that the onset of the supercritical branch is independent of $R_{\mathrm{v}}$ and that, near the bifurcation point, the slope of the supercritical branch is also independent of $R_{\mathrm{v}}$. On the supercritical branch the streamfunction is negative in the center of the cavity and the flow therefore consists (Fig. 7) of a dominant counterclockwise rotating cell with two identical recirculations in the upper right and lower left corners. The calculation also confirms the relation $T^{\prime}=\mathrm{Le} C^{\prime}$ between the thermal and concentration perturbations in the neighborhood of the bifurcation point. Note that the streamfunction has point symmetry while the thermal and solutal perturbations are antisymmetric (Fig. 7). Such a solution is invariant under the symmetry $S$ as predicted by the linear stability analysis. The subcritical regime extends down to much lower convective thresholds $\left(\mathrm{Gr}=676\right.$ for $\left.R_{\mathrm{v}}=0\right)$ as reported by Ghorayeb and Mojtabi (1997) and terminates in a saddle-node bifurcation on the finite amplitude branch. As $R_{\mathrm{v}}$ increases, the value of $\mathrm{Gr}$ at this turning point decreases continuously and in zero gravity motion can exist for all values of $R_{\mathrm{v}}>2860$, in agreement with Fig. 4. The flow structure is qualitatively as shown in Fig. 5, i.e., it consists of a single clockwise roll. Fig. 6 shows that near Gr $\approx 1230$ the convection amplitude is almost independent of the vibration strength. It is worth mentioning that the supercritical solution is obtained easily only if initial data with (nearly) the right symmetry properties are prescribed; otherwise the solution evolves towards the subcritical solution which appears to have a much larger domain of attraction.

Direct simulations of the system (3)-(6) have also been carried out. The solution of the NavierStokes equation with a time-dependent force per unit volume requires a substantially longer computation time in comparison with the mean-flow equations and the same resolution requirements. The results of the latter calculations are recovered for all $\hat{f}=2 \pi \hat{\omega}>200 \mathrm{~Hz}(f \gg 1 \mathrm{~Hz}$ for water at $20^{\circ} \mathrm{C}$ and $\left.L=1 \mathrm{~cm}\right)$. The value $\hat{f}^{*} \approx 200$ corresponds to the limit of the validity of the mean field treatment for our problem. Of course, as shown in Fig. 8 all of the variables now depend on time but their mean values over the vibration period are independent of the frequency whenever $\hat{f}>\hat{f}^{*}$. 


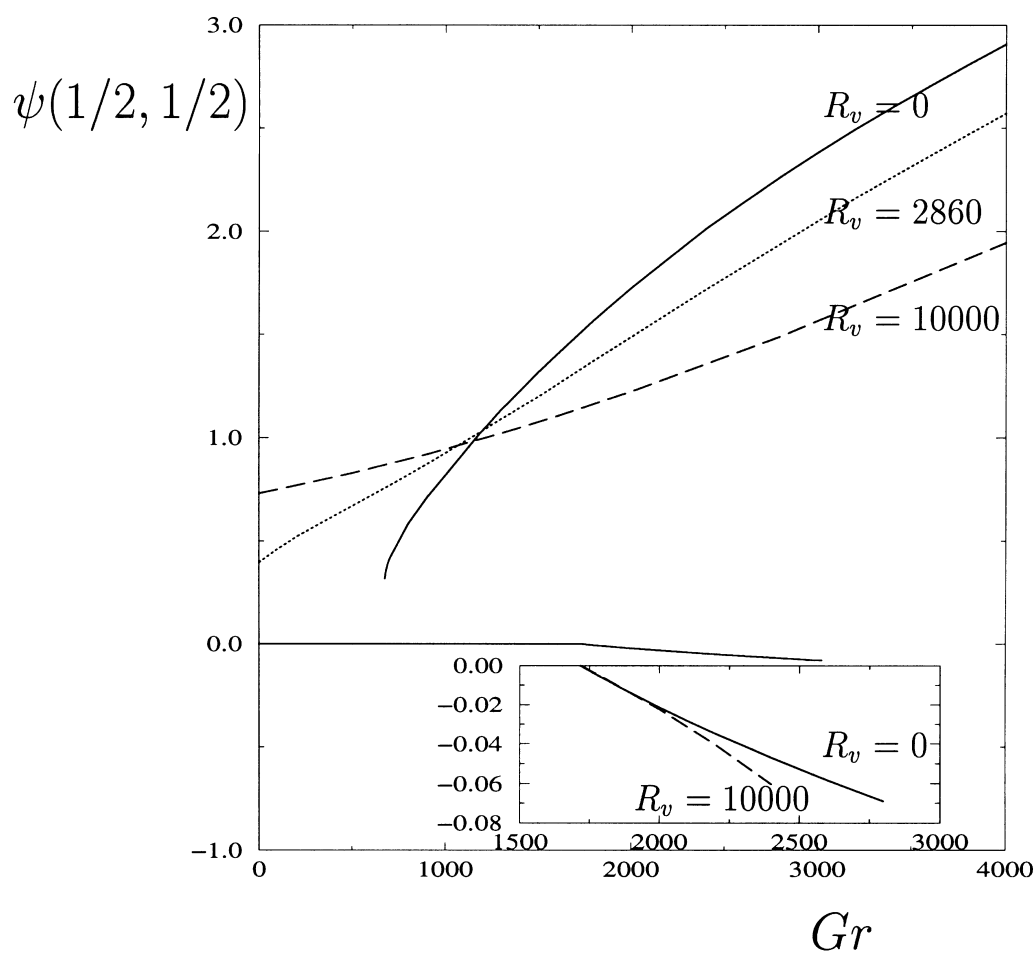

Fig. 6. Bifurcation diagram in the $\psi\left(\frac{1}{2}, \frac{1}{2}\right)-\mathrm{Gr}$ plane for horizontal vibration and different values of $R_{\mathrm{v}}$ when Le $=11$, $\operatorname{Pr}=1$. The saddle-node bifurcation moves from $\mathrm{Gr}=676$ (for $R_{\mathrm{v}}=0$ ) to $\mathrm{Gr}=0$ (for $R_{\mathrm{V}}=2860$ ). The inset is an enlargement of the supercritical branch for different values of $R_{\mathrm{v}}$. The vibration strength $\left(R_{\mathrm{v}}\right)$ does not affect the transcritical branch in the neighborhood of the primary bifurcation. Resolution is $25 \times 25$.

In this example, the value of the mean Nusselt number $\mathrm{Nu}$ is 1.1775 for $\hat{f}=100,1.1758$ for $\hat{f}=300$ and 1.1756 for $\hat{f}=500$, i.e., $\mathrm{Nu}$ varies by less than $0.5 \%$ over this range of frequencies. For slower oscillations resonance phenomena may occur but these are beyond the scope of the present work.

Vibration at $\alpha=\frac{\pi}{4}$ : For vibration at $45^{\circ}$ the supercritical branch is present for $\mathrm{Gr}>1715$ and its slope decreases (in magnitude) with increasing $R_{\mathrm{v}}$ (Fig. 9), as predicted by the weakly nonlinear analysis. In the limiting case $R_{\mathrm{v}} \rightarrow \infty$, the supercritical branch becomes a purely diffusive one. The subcritical branch behaves in much the same way as in the previous case. On this branch, the streamfunction is positive in the center of the cavity and the flow regime resembles that in Fig. 5, but with the vibration-independent solution moved to $\mathrm{Gr} \approx 676$. Note that this value corresponds to the turning point on the subcritical branch in the absence of vibration. In zero gravity $(\mathrm{Gr}=0)$ thermo-vibrational convection can be produced for $R_{\mathrm{v}}>5400$.

Vertical vibration $\left(\alpha=\frac{\pi}{2}\right)$ : For vertical vibration the numerical results also confirm that the value of the critical Grashof number $(\mathrm{Gr}=1715$ when $\mathrm{Le}=11)$ is independent of the vibrational Rayleigh number (see Fig. 10). The motion on the supercritical branch is a three-cell flow with the dominant cell being counterclockwise. The isolines of the temperature and concentration are almost parallel and vertical, and are consistent with the relation $T^{\prime}=\mathrm{Le} C^{\prime}$ found in weakly nonlinear theory. As $R_{\mathrm{v}}$ increases the slope of the transcritical branch becomes steeper and steeper and for $R_{\mathrm{v}}>13000$ it 

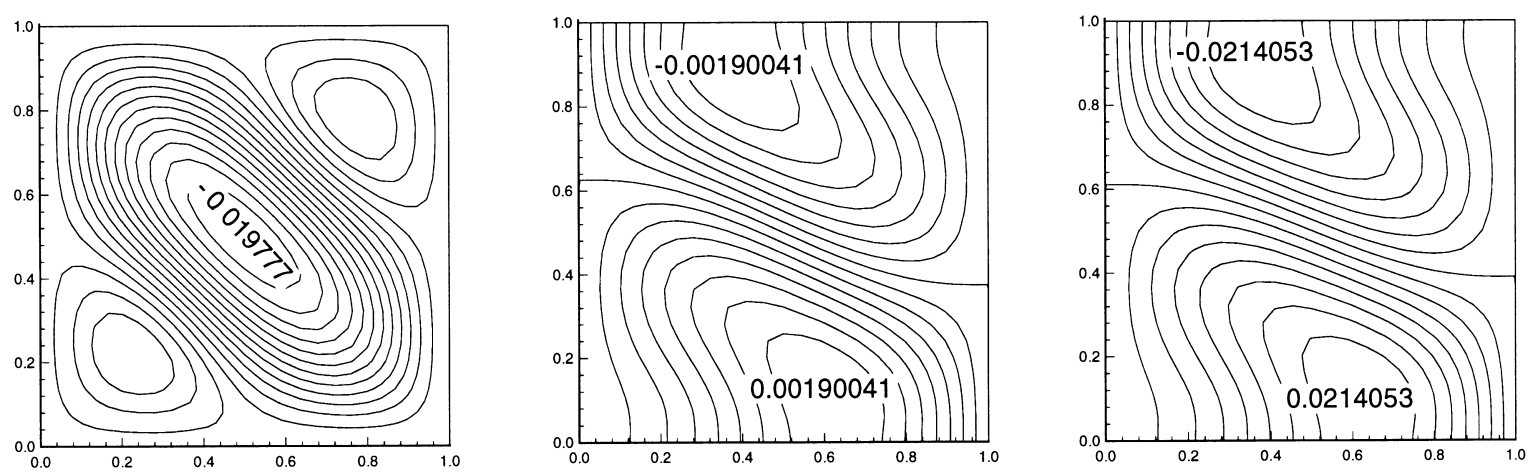

Fig. 7. Streamlines, and lines of constant temperature and concentration perturbations for $\mathrm{Gr}=2000$ and $R_{\mathrm{v}}=10000$ and horizontal vibration ( $\mathrm{Le}=11, \operatorname{Pr}=1, A=1$ ). Resolution is $25 \times 25$.

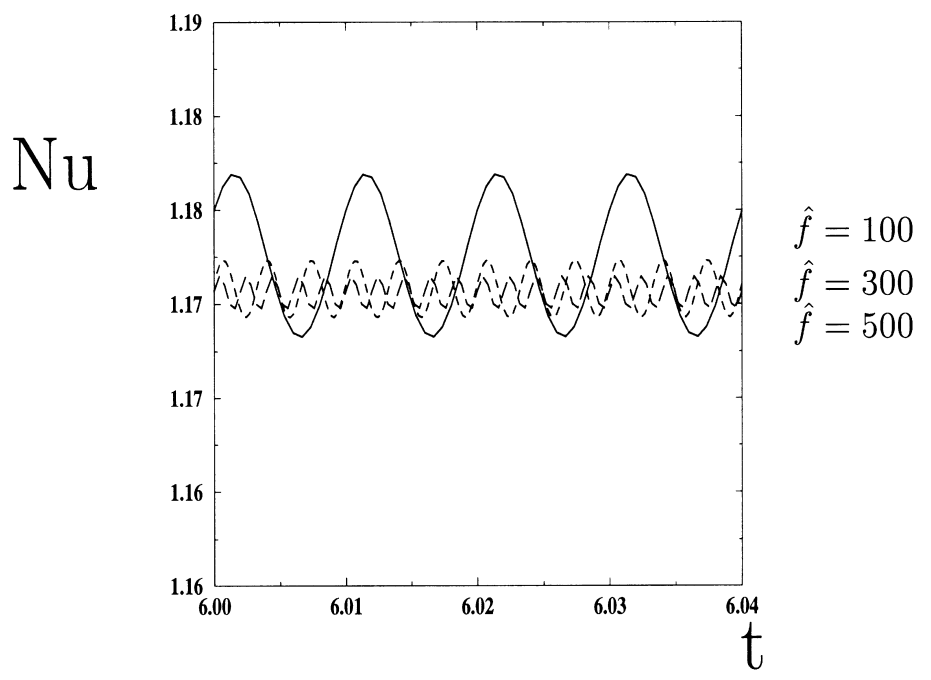

Fig. 8. The Nusselt number $\mathrm{Nu}$ as a function of the dimensionless time $t$ for $\hat{f}=100$ (solid line), $\hat{f}=300$ (dotted line) and $\hat{f}=500$ (long-dashed line), computed from the primitive equations for $\operatorname{Pr}=1, \mathrm{Le}=11, A=1, \mathrm{Gr}=2000$ and $R_{\mathrm{v}}=2000$. Resolution is $33 \times 33$.

changes sign. In this regime there is a stable small amplitude supercritical branch with $\psi>0$ which soon loses stability at a saddle-node bifurcation at which the branch turns around towards smaller Gr joining the original stable small amplitude $\psi>0$ subcritical branch at a second saddle-node bifurcation. This behavior can be established by analyzing the degenerate transcritical bifurcation in which the coefficient of the quadratic term vanishes, as described in Section 4.2. This second

$>0$ branch in turn loses stability at a further saddle-node bifurcation at the right where it turns to the left and joins the large amplitude subcritical branch (see Fig. 10); the saddle-node on the large amplitude subcritical branch moves towards higher Grashof numbers with increasing vibrational Rayleigh number. For $\mathrm{Gr}=1600$ and $R_{\mathrm{v}}=50000$, the values of the Nusselt number $\mathrm{Nu}$ and the 


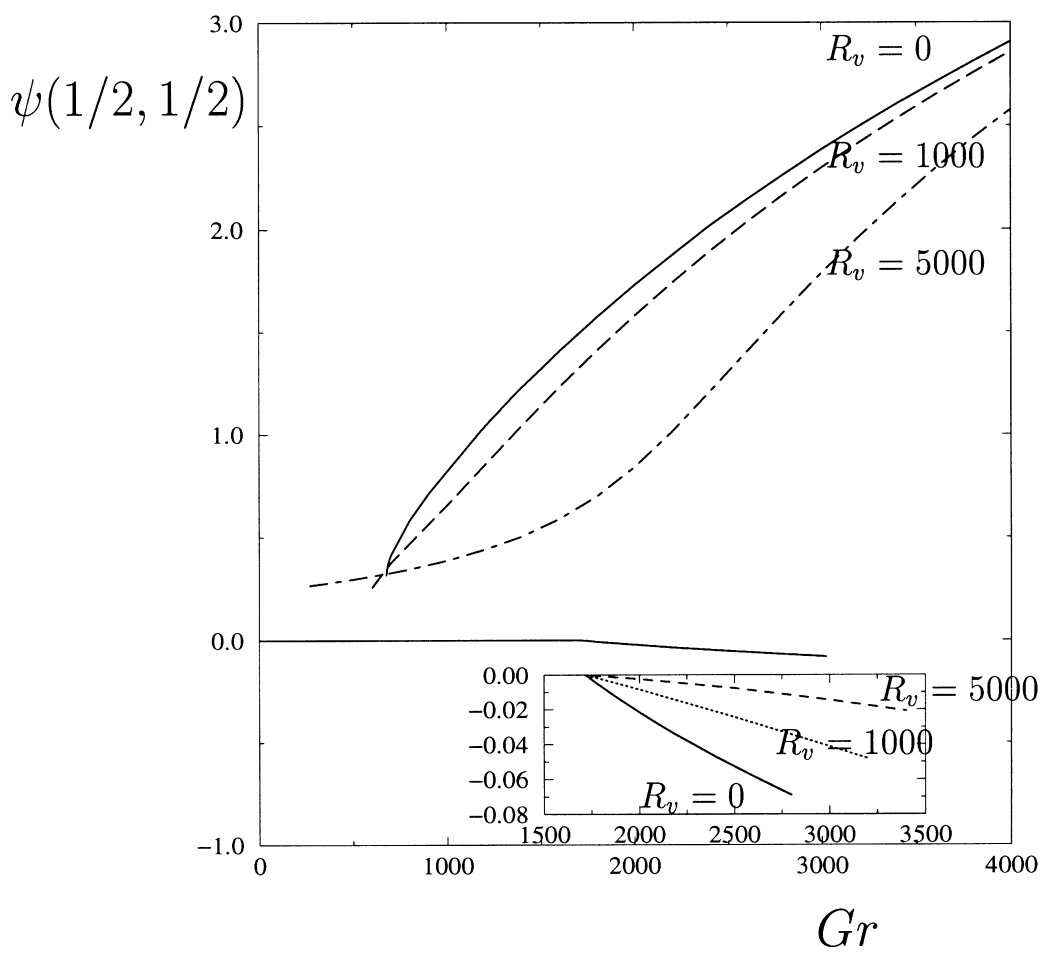

Fig. 9. Bifurcation diagram in the $\psi\left(\frac{1}{2}, \frac{1}{2}\right)-$ Gr plane for vibration at $\alpha=\frac{\pi}{4}$ and different values of $R_{\mathrm{v}}$ when Le $=11$, $\operatorname{Pr}=1$. The inset is an enlargement of the transcritical branch of solutions for different values of $R_{\mathrm{v}}$. The slope of this supercritical branch decreases (in modulus) as $R_{\mathrm{v}}$ increases. Resolution is $25 \times 25$.

corresponding dimensionless number describing the solute flux (the Sherwood number Sh) on the lower $\psi>0$ branch are, respectively, 1.004 and 1.397; note that these do not satisfy the expected relation $\mathrm{Nu}-1=\operatorname{Le}^{2}(\mathrm{Sh}-1)$, see Bardan et al. (2000). The corresponding flow structure is shown in Fig. 11. A finite amplitude $\psi<0$ branch is also present and plotted for $R_{\mathrm{v}}=20000$ in the enlargement. In contrast, the flow on the large amplitude branch consists of a clockwise single cell flow. The critical Grashof number at which a finite amplitude solution first appears depends on $R_{\mathrm{v}}$ and decreases gradually with increasing $R_{\mathrm{v}}$, becoming zero when $R_{\mathrm{v}}$ exceeds 50000 . In this case a finite amplitude vibration-driven state coexists with a stable conduction state in zero gravity.

We have examined one additional (and more realistic) value of the Lewis number. For Le $=$ 51, corresponding to oxygen in water, the critical Grashof number is 343. The primary bifurcation remains transcritical and the effect of vibration is qualitatively similar to that at Le $=11$. However, the bifurcation diagram for vertical vibration shown in Fig. 12 reveals the presence of a new feature. The transcritical branch for $R_{\mathrm{v}}=2000$ consists of a three-cell supercritical branch with a counterclockwise central cell; there is also a subcritical solution branch consisting of a small but finite amplitude three-cell flow with a clockwise central cell. Once again we presume that this branch is connected to the transcritical bifurcation via an unstable subcritical branch. The branch itself loses stability with increasing $\mathrm{Gr}$ at a saddle-node bifurcation providing a connection to the large amplitude single clockwise cell states via another unstable segment of the subcritical branch. It is along 


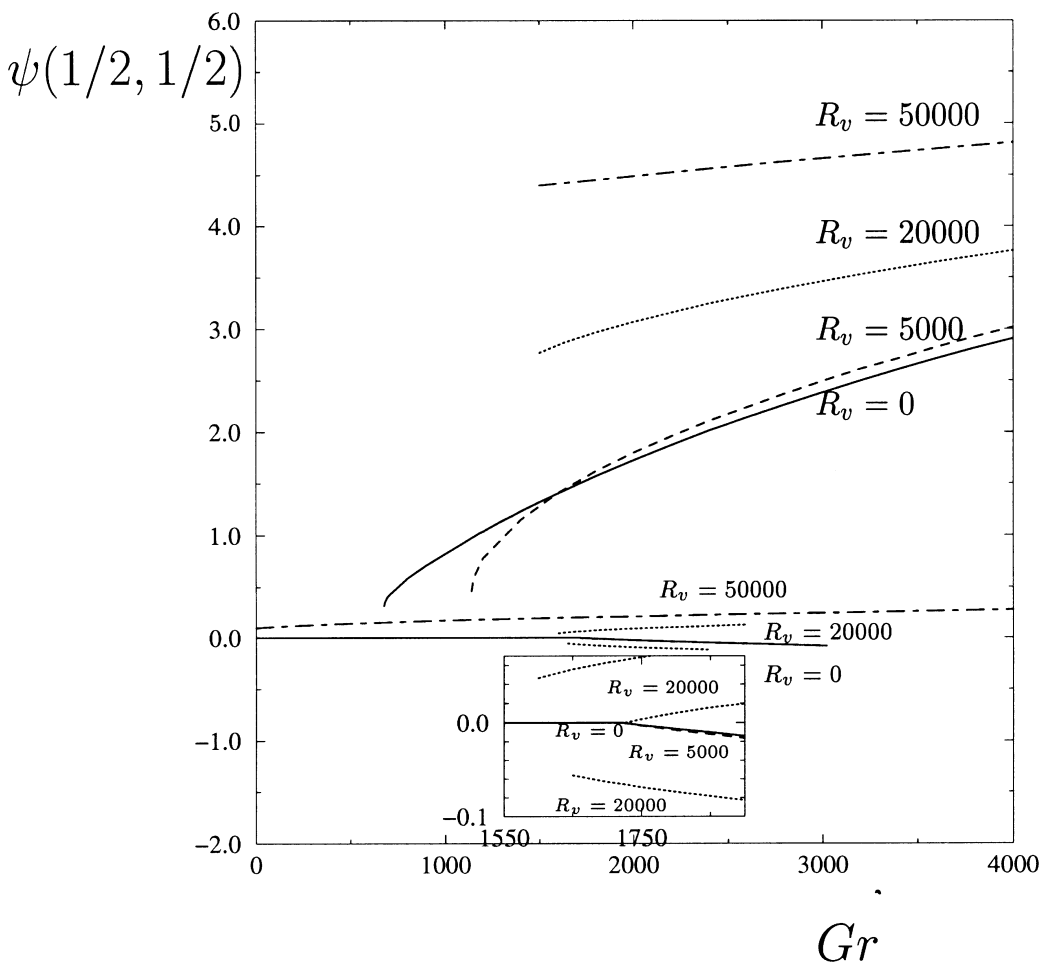

Fig. 10. Bifurcation diagram in the $\psi\left(\frac{1}{2}, \frac{1}{2}\right)-\mathrm{Gr}$ plane for vertical vibration and different values of $R_{\mathrm{v}}$ when $\mathrm{Le}=11$, $\operatorname{Pr}=1$. The saddle-node bifurcation moves from $\mathrm{Gr}=676$ (for $\left.R_{\mathrm{v}}=0\right)$ to $\mathrm{Gr}=1620$ (for $R_{\mathrm{v}}=20000$ ). The inset is an enlargement of the supercritical branch for different values of $R_{\mathrm{v}}$. The slope of this transcritical branch increases (in modulus) as $R_{\mathrm{v}}$ increases and the branch becomes subcritical for $R_{\mathrm{v}}>13000$. At $R_{\mathrm{v}}=20000$ there are as many as four stable branches for $\mathrm{Gr}>\mathrm{Gr}^{(0)}$, three at small amplitude and a fourth large amplitude branch, separated by unstable branches. For $R_{\mathrm{v}}=50000$ stable convection is possible even when $\mathrm{Gr}=0$. Resolution is $25 \times 25$.
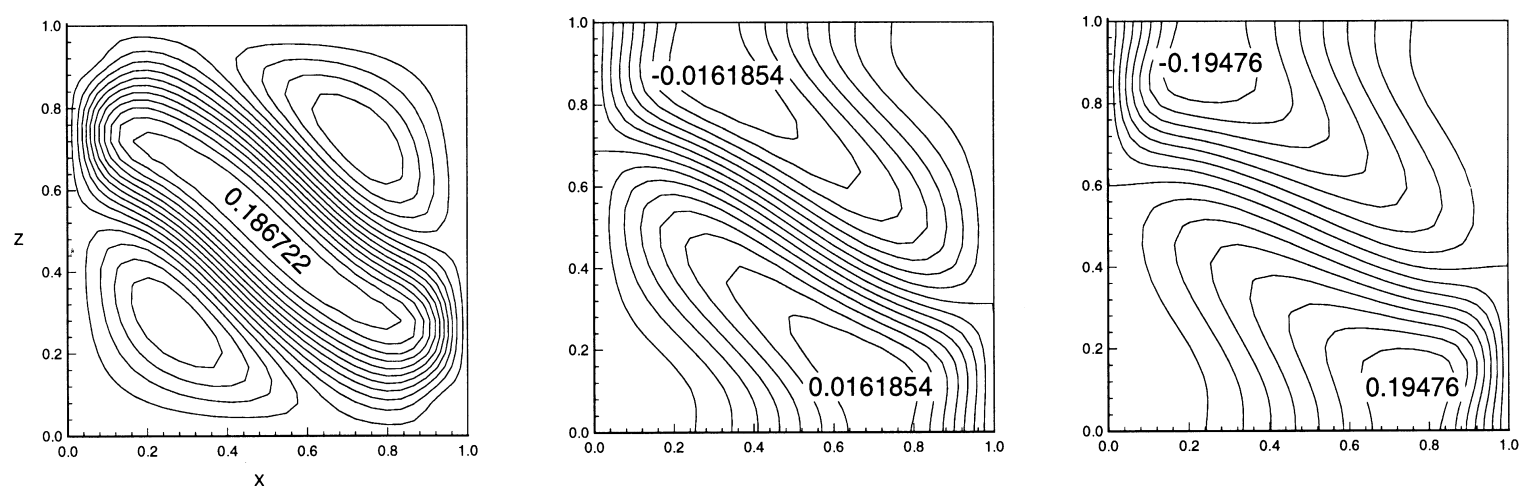

Fig. 11. Lines of constant streamfunction, temperature and concentration perturbations for $\mathrm{Gr}=1600, R_{\mathrm{v}}=50000$ and vertical vibration $(\mathrm{Le}=11, \operatorname{Pr}=1, A=1)$. Resolution is $25 \times 25$. 


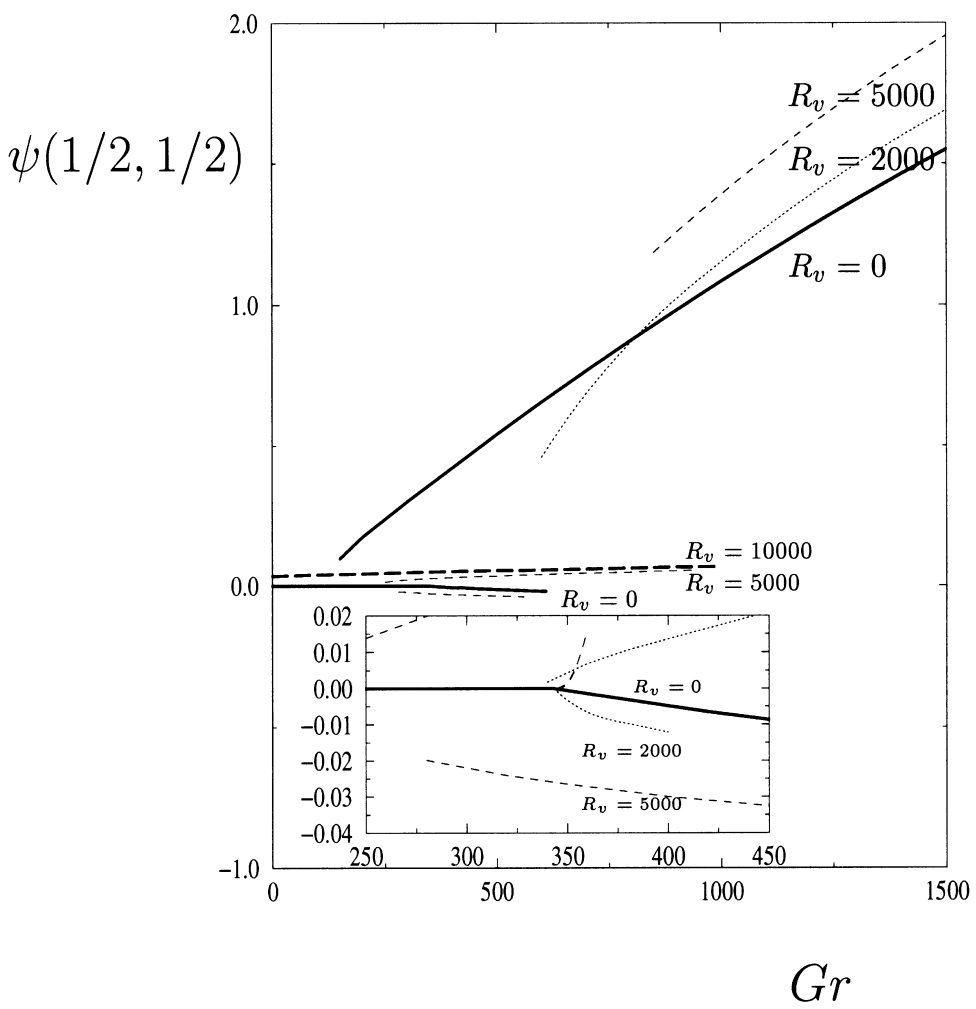

Fig. 12. Bifurcation diagram in the $\psi\left(\frac{1}{2}, \frac{1}{2}\right)-\mathrm{Gr}$ plane for vertical vibration and different values of $R_{\mathrm{v}}$ when Le $=51$, $\operatorname{Pr}=1$, for comparison with Fig. 10. Resolution is $35 \times 35$.

this unstable segment that the form of the solution gradually changes from the three cell state to the single cell state as the central clockwise cell grows and the counterclockwise corner cells shrink. Such a transition must occur since the single cell state cannot connect to the three cell states present on either side of the transcritical bifurcation. As a result there is now an interval of Grashof numbers with three stable coexisting solutions. It is also noteworthy that for $R_{\mathrm{v}}>2600$ the transcritical branch again changes direction (see Fig. 12), generating an additional wiggle in the $\psi>0$ branch, i.e., there are now three stable segments on the $\psi>0$ branch instead of two. At the same time the $\psi<0$ branch develops a subcritical segment. As a result the small amplitude $\psi<0$ solutions become unstable although they once again acquire stability at a secondary saddle-node bifurcation. Altogether there are now four stable solution segments (cf. Fig. 12 for $R_{\mathrm{v}}=5000$ ). Once again these results follow from the unfolding of the degenerate transcritical bifurcation. Our numerical simulations indicate that the value of $R_{\mathrm{v}}$ at which this change takes place is inversely proportional to Le when the Prandtl number remains fixed. Fig. 13 shows the development of solutal boundary layers on the large amplitude $\psi>0$ branch with increasing Le, as well as the constant concentration core produced by the expulsion of concentration gradients from the convective cell that occurs at these amplitudes. 

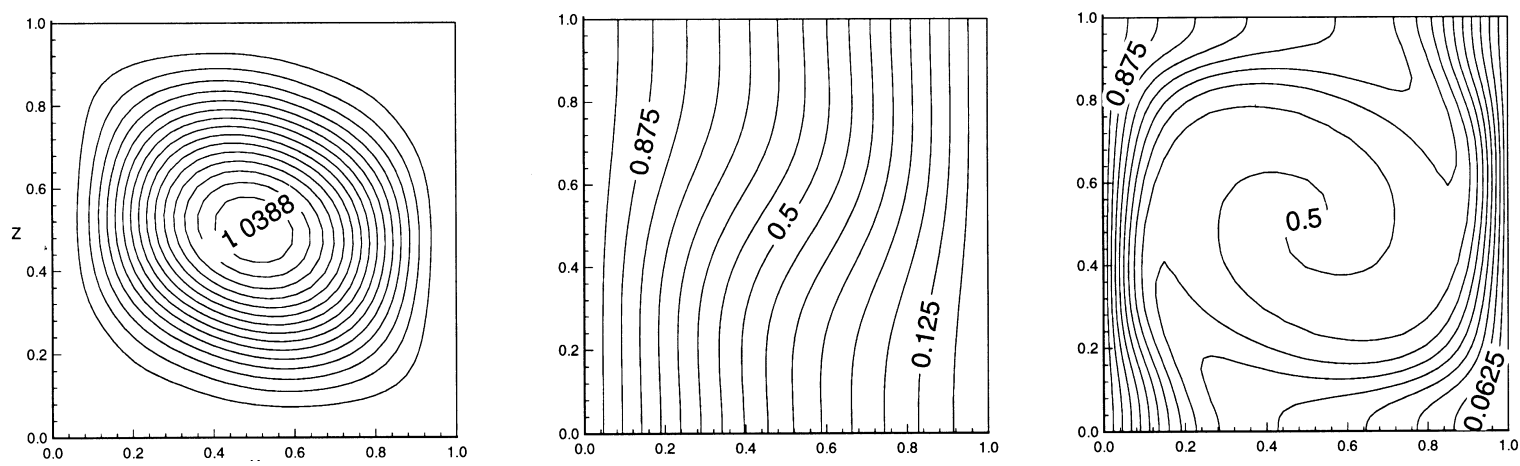

Fig. 13. Streamlines, isotherms and isoconcentrations for $R_{\mathrm{v}}=5000, \mathrm{Gr}=850$ and vertical vibration when $\mathrm{Le}=51, \mathrm{Pr}=1$. Resolution is $35 \times 35$.

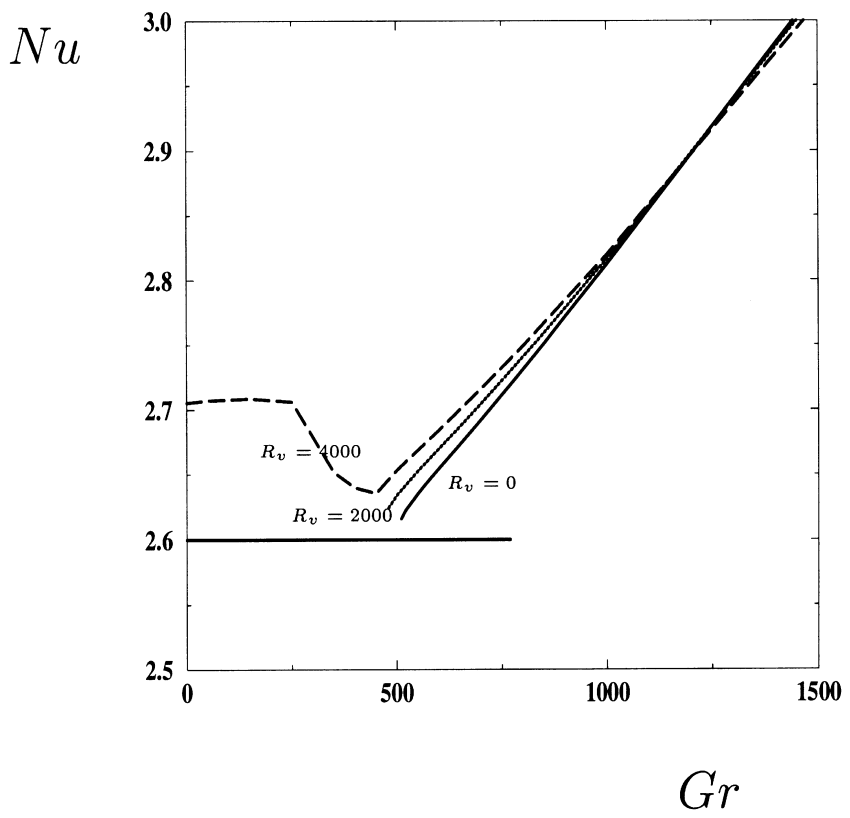

Fig. 14. Bifurcation diagram in the $\mathrm{Nu}-\mathrm{Gr}$ plane for horizontal vibration and different values of $R_{\mathrm{V}}$ when $\mathrm{Le}=11$, $\mathrm{Pr}=1$, $A=2.6$, and $R_{\mathrm{v}}=0$ (solid line), $R_{\mathrm{v}}=2000$ (dotted line) and $R_{\mathrm{v}}=4000$ (long-dashed line). Resolution is $33 \times 33$.

\subsubsection{Interaction between gravity and vibration driven convection when $A=2.6$}

In this section we briefly summarize the corresponding results for $A=2.6$. In this case the primary instability leads to an antisymmetric eigenmode and the bifurcation is therefore a pitchfork. The resulting bifurcation diagram for horizontal vibration $(\alpha=0)$ is shown in Fig. 14. As already mentioned the pitchfork for $R_{\mathrm{v}}=0$ is subcritical. The initially unstable convection branch acquires stability at a saddle-node bifurcation at which the branch turns towards increasing Gr. The figure shows that the location of this saddle-node bifurcation moves towards smaller $\mathrm{Gr}$ as $R_{\mathrm{v}}$ increases and 

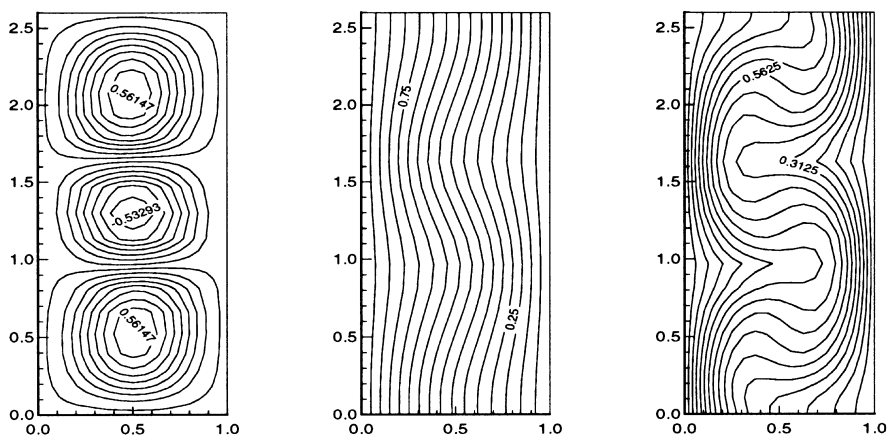

$$
G r=100
$$
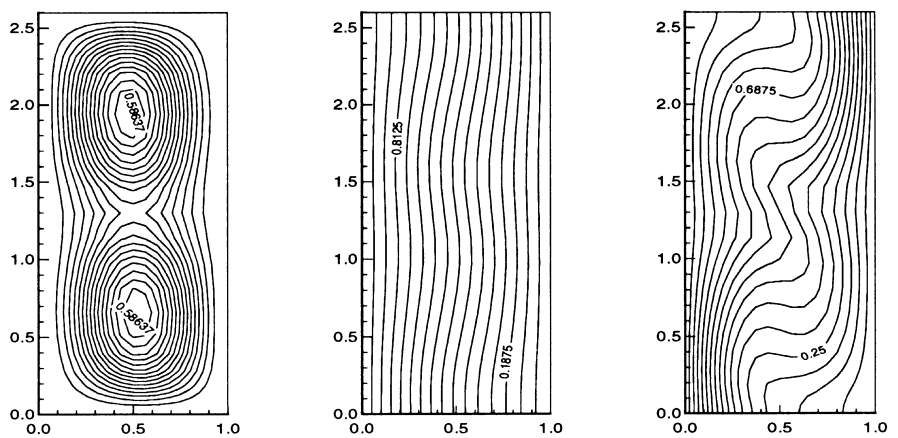

$$
G r=400
$$
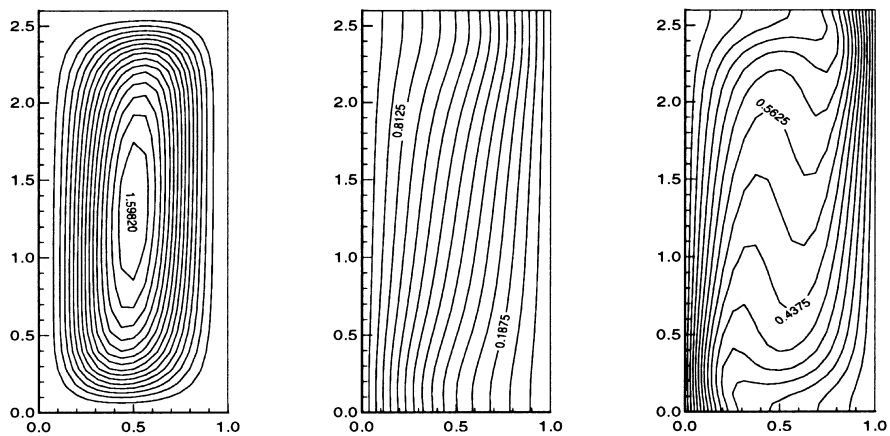

$G r=1000$

Fig. 15. Streamlines, isotherms and isoconcentrations for horizontal vibration and $R_{\mathrm{v}}=4000$ when $\mathrm{Le}=11, \operatorname{Pr}=1$ and $A=2.6$. Resolution is $33 \times 33$.

that for sufficiently large $R_{\mathrm{v}}$ stable finite amplitude convection coexists with the (stable) conduction state even in zero gravity $(\mathrm{Gr}=0)$. This is so for the case illustrated in Fig. 15; this figure shows the streamlines, isotherms and isoconcentration lines for $R_{\mathrm{v}}=4000$ and several values of Gr on the subcritical branch. For small values of $\mathrm{Gr}$ the flow consists of three cells of which the central one is counterclockwise while the two stronger outer cells are clockwise. By $\mathrm{Gr}=400$ the central cell has shrunk to nothing and for larger values of Gr the flow consists of a single large clockwise cell filling the entire cavity. Note that these solutions are all symmetric with respect to the symmetry 


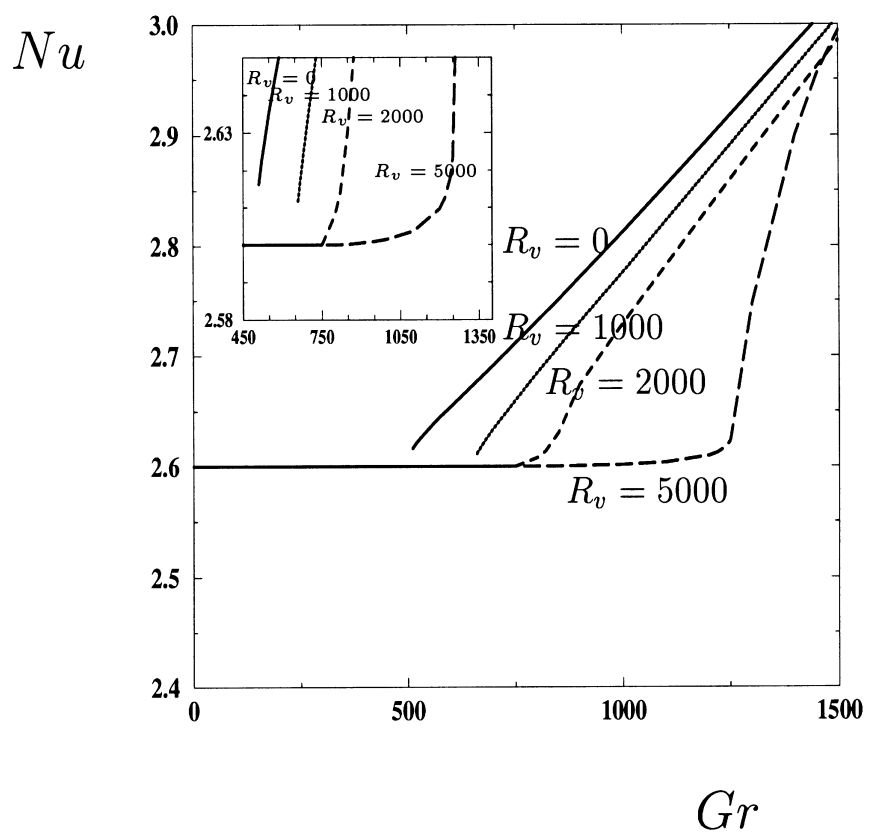

Fig. 16. Bifurcation diagram in the $\mathrm{Nu}-\mathrm{Gr}$ plane for vertical vibration and different values of $R_{\mathrm{v}}$ when Le $=11$, $\operatorname{Pr}=1$, $A=2.6$, and $R_{\mathrm{v}}=0$ (solid line), $R_{\mathrm{V}}=1000$ (dotted line), $R_{\mathrm{V}}=2000$ (dashed line) and $R_{\mathrm{V}}=5000$ (long-dashed line). Resolution is $33 \times 33$.

operation $S$. Such solutions cannot be connected to the original pitchfork bifurcation. Consequently, a symmetry-breaking bifurcation must occur along the solution branch as this is followed to smaller and smaller amplitudes, and it is these states that develop into the antisymmetric states born in the primary pitchfork bifurcation. In the present case these states are unstable.

For vertical vibration $\left(\alpha=\frac{\pi}{2}\right)$ the situation is markedly different. In this case, as shown in Fig. 16, increasing $R_{\mathrm{v}}$ decreases the subcriticality of the pitchfork; for sufficiently large $R_{\mathrm{v}}$ this bifurcation becomes supercritical and stable solutions can be found arbitrarily close to onset of the primary instability. Fig. 17 shows the evolution of the flow with decreasing Gr when $R_{\mathrm{v}}=5000$. At large Gr the flow is again symmetric and consists of two strong cells both of which are clockwise. As Gr decreases these two cells merge into a single clockwise cell, leaving weaker counterclockwise cells in the top right and bottom left corners, but the overall symmetry with respect to $S$ remains. However, as Gr decreases further, this symmetry is lost; we show the resulting solution at $\mathrm{Gr}=795$. By $\mathrm{Gr}=770$ the solution is very weak but almost entirely antisymmetric; this solution connects to the pitchfork bifurcation which for these parameter values occurs at $\mathrm{Gr}=765$.

\section{Conclusion}

We have described a theoretical and numerical study of doubly diffusive convection in square and rectangular cavities in the special case permitting the existence of an equilibrium or quasi-equilibrium 

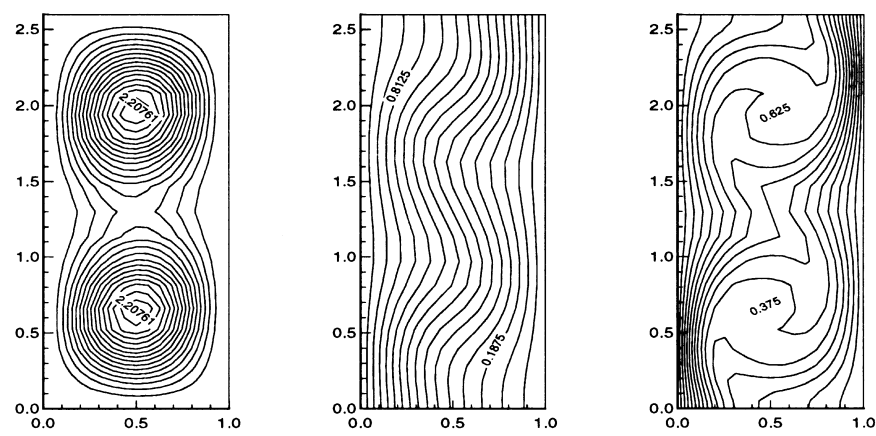

$$
G r=1680
$$
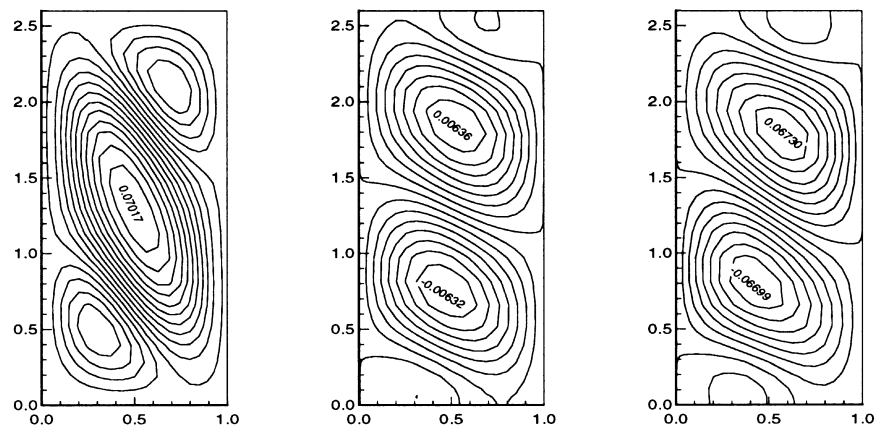

$$
G r=910
$$
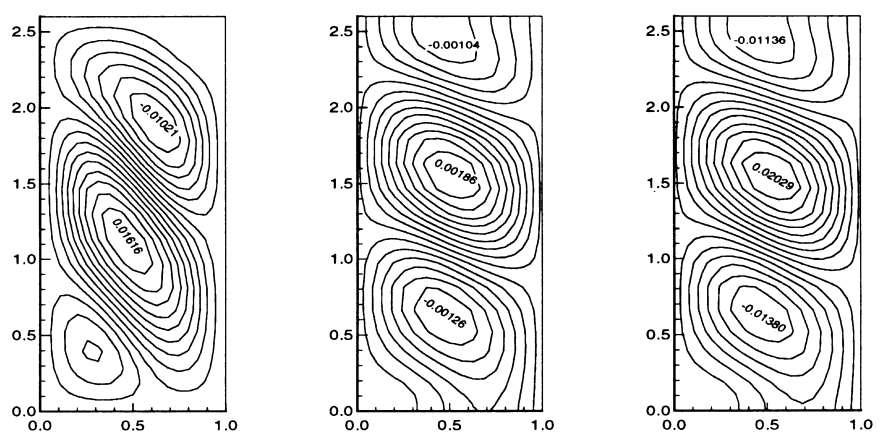

$$
G r=795
$$
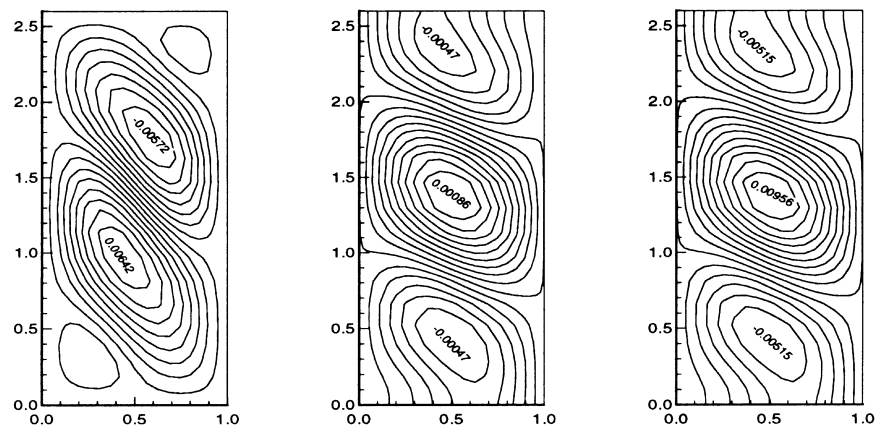

$$
G r=770
$$

Fig. 17. Streamlines, isotherms and isoconcentrations for $R_{\mathrm{v}}=5000$ and vertical vibration when $\mathrm{Le}=11, \operatorname{Pr}=1, A=2.6$ and $\mathrm{Gr}=1680$. For $\mathrm{Gr}=770, \mathrm{Gr}=795$ and $\mathrm{Gr}=910$ the contours of constant streamfunction, temperature and concentration perturbations are shown instead. Resolution is $33 \times 33$. 
state. We explored the effects of rapid vibration on this system as a function of both strength and direction. A close agreement between the theoretical and numerical results was found. In zero gravity convective motion appears in a square cavity with $\mathrm{Le}=11$ for a suitably chosen vibration axis whenever the vibration strength exceeds $R_{\mathrm{v}}=2610$. The range of orientations for which instability occurs increases with $R_{\mathrm{v}}$, with an upper limit set by $\alpha=73^{\circ}$; for larger angles the conduction solution (i.e., the equilibrium state) is unconditionally stable. The interaction between static gravity and vibration-driven convection is similar to that found for the static case. When $A=1$ the primary bifurcation is transcritical and when $\alpha=0$ and $\alpha=\frac{\pi}{4}$ leads to a stable supercritical branch and a stable finite amplitude subcritical branch. In contrast for vertical vibration there may be several subcritical branches connected by unstable solutions, provided the vibrational Rayleigh number is sufficiently high. On the small amplitude supercritical branch both horizontal and vertical vibration increases the strength of convective motion, while the opposite is the case when $\alpha=\frac{\pi}{4}$. In all cases sufficiently high $R_{\mathrm{v}}$ leads to bistability between the conduction state and vibration-driven convection in zero gravity. When $A=2.6$ horizontal vibration is very effective in driving thermo-vibrational convection; in contrast vertical vibration suppresses thermo-vibrational convection effectively, and substantial buoyancy forces are required to sustain fluid motion. This observation suggests a possible protocol that could be used to control convective motion induced by stray gravitational fields or DC accelerations.

The reason different vibration directions have such different effect can be understood by considering the direction of the effective "vibration force" due to the oscillation of the cavity. This force is described by the new term on the right side of the Navier-Stokes equation (12) and represents the tendency of the fluid to flow (on average) along gradients of density inhomogeneities (cf. Gershuni and Lyubimov, 1998). This flow is in addition to the doubly diffusive flows present in the absence of vibration. When $N=-1$ this force is proportional to $\operatorname{Gr}(\nabla T-\nabla C)$; since for typical parameter values the concentration gradients in the stationary flow are stronger than those in the temperature the direction of the vibrational force is determined primarily by the former. For example, the isoconcentration contours shown in Fig. 13 for vertical vibration indicate that the overall vibration force in the left half of the cavity is towards to the lower left and conversely in the right half of the cavity. Thus the vibration-induced flow is counterclockwise and hence opposite to the doubly diffusive flow. As a consequence, increasing $R_{\mathrm{v}}$ reduces the overall circulation and the saddle-node bifurcation on the subcritical branch therefore moves towards higher values of Gr. This conclusion agrees with the results shown in Fig. 12. A similar conclusion holds for the flow shown in Fig. 17 at $\mathrm{Gr}=1680$ and explains why the pitchfork bifurcation for $A=2.6$ becomes supercritical for sufficiently large values of $R_{\mathrm{v}}$. The vibration-induced counterclockwise circulation weakens with decreasing $\mathrm{Gr}$, however, and close to the primary pitchfork bifurcation the linear relation $T^{\prime}=\operatorname{Le} C^{\prime}$ indicates that the direction of the vibration-induced flow is determined instead by the temperature field. However, the vibration-induced flow remains in the same direction as the doubly diffusive flow. Similarly, an examination of the contours of constant concentration for horizontal vibration (Fig. 15) shows that at $\mathrm{Gr}=100$ the vibration-induced circulation will be clockwise at the top and bottom of the cavity and counterclockwise in the center. It follows that in this case the vibration-induced flow is in the same direction as the overall flow and hence that increasing $R_{\mathrm{v}}$ can only strengthen the observed circulation. As a result the saddle-node bifurcation on the subcritical branch moves towards lower values of the Grashof number as $R_{\mathrm{v}}$ increases, as found in Fig. 14. In this case thermo-vibrational convection can be driven even under zero gravity conditions. 
In certain cases we have seen that the direction of the transcritical bifurcation changes with increasing $R_{\mathrm{v}}$, producing a wiggle in the subcritical branch and increasing the multistability of the system. It appears likely that each time the transcritical bifurcation changes direction an additional wiggle is introduced on the subcritical branch, resulting ultimately in a steady-state branch with a large number of left-right oscillations as in the related problem studied by Tsitverblit (1995).

\section{Acknowledgements}

This work would not have been possible without the support of C.N.E.S. (Centre National d'Etudes Spatiales) and Université Paul Sabatier.

\section{References}

Alavyoon, F., 1994. On natural convection in vertical porous enclosures due to opposing fluxes of heat and mass prescribed at the vertical walls. Int. J. Heat Mass Transfer 37, 195-206.

Alexander, J.I.D., 1990. Low gravity experiment sensitivity to residual acceleration: a review. Microgravity Sci. Technol. III 2, 52-68.

Alexander, J.I.D., Amiroudine, S., Ouazzani, J., Rosenberg, J., 1991. Analysis of low gravity tolerance of BridgmanStockbarger crystal growth II. Transient and periodic accelerations. J. Crystal Growth 113, 21-38.

Azaiez, M., Bernardi, C., Grundmann, M., 1994. Spectral methods applied to porous media. East-West J. Numer. Math. 2, 91-105.

Bardan, G., Bergeon, A., Knobloch, E., Mojtabi, A., 2000. Nonlinear doubly diffusive convection in vertical enclosures. Physica D 138, 91-113.

Belyaev, M.Yu., Zykov, S.G., Ryabukha, S.B., Sazonov, V.V., Sarychev, V.A., Stazhkov, V.M., 1994. Mathematical modelling and measurements on the orbital station Mir. Mech. Zhidk Gasa 5, 5-15.

Birigen, S., Peltier, L.J., 1990. Numerical simulation of 3-D Bénard convection with gravitational modulation. Phys. Fluids 2, 754-764.

Braverman, L.M., 1987. On vibrational convective instability of plane fluid layer in weightlessness. Ural Branch Acad. Sci. SSSR 29, 29-35.

Canuto, C., Hussaini, M.Y., Quarteroni, A., Zang, T.A., 1987. Spectral Methods in Fluid Dynamics. Springer, New York.

Chang, J., Lin, T.F., 1993. Unsteady thermosolutal opposing convection of liquid-water mixture in a square cavity-II. Flow structure and fluctuation analysis. Int. J. Heat Mass Transfer 36, 1333-1348.

Chernatynsky, A.N., Gershuni, G.Z., Monti, R., 1995. Transient regimes of thermovibrational convection in a closed cavity. Microgravity Quart. 3, 55-67.

Crawford, J.D., Knobloch, E., 1991. Symmetry and symmetry-breaking bifurcation in fluid dynamics, Annu. Rev. Fluid Mech. 23, 341-87.

Gershuni, G.Z., Kolesnikov, A.K., Legros, J.-C., Myznikova, B.I., 1997. On the vibrational convective instability of a horizontal binary-mixture layer with Soret effect. J. Fluid Mech. 330, 251-269.

Gershuni, G.Z., Lyubimov, D.V., 1998. Thermal Vibrational Convection. Wiley, New York.

Gershuni, G.Z., Zhukhovitsky, E.M., 1979. On free convection in vibrational field in weightlessness. Dokl. Akad. Nauk SSSR 294, 55-67.

Gershuni, G.Z., Zhukhovitsky, E.M., 1981. On convective instability of fluid in vibrational field in weightlessness. Izv. Akad. Nauk SSSR Mekh. Zhidk. Gaza 4, 12-19.

Gershuni, G.Z., Zhukhovitsky, E.M., 1988. On convective instability of a convective flow with respect to 3D-disturbances. Izv. Akad. Nauk SSSR Mekh. Zhidk. Gaza 2, 116-122.

Ghorhayeb, K., Mojtabi, A., 1997. Double diffusive convection in a vertical rectangular cavity. Phys. Fluids 9, $2339-2348$.

Gobin, D., Bennacer, R., 1994. Double diffusion in a vertical fluid layer: onset of the convection regime. Phys. Fluids 6, $59-67$. 
Golubitsky, M., Schaeffer, D.G., 1985. Singularities and Groups in Bifurcation Theory, Vol. 1. Springer, New York.

Gresho, P.M., Sani, R.L., 1970. The effects of gravity modulation on the stability of a heated fluid layer. J. Fluid Mech. 40, 783-806.

Guckenheimer, J., Holmes, P., 1983. Nonlinear Oscillations, Dynamical Systems and Bifurcations of Vector Fields. Springer, New York.

Khallouf, H., 1995. Simulation numérique de la convection thermo-vibrationnelle par une méthode spectrale. These de doctorat de l'université Paul Sabatier, Toulouse III.

Khallouf, H., Gershuni, Z.G., Mojtabi, A., 1995. Numerical study of two-dimensional thermovibrational convection in rectangular cavities. Numer. Heat Transfer 27, 297-305.

Krishnan, R., 1989. A numerical study of the instability of double diffusive convection in a square enclosure with a horizontal temperature and concentration gradients. ASME National Heat Transfer Conference, Philadelphia, pp. 357-368.

Landau, L.D., Lifshitz, E.M., 1988. Mechanics. ASME Nauka, Moscow (in Russian).

Murray, B.T., Coriell, S.R., McFadden, G.B., 1991. The effect of gravity modulation on solutal convection during directional solidification. J. Crystal Growth 110, 713-723.

Nelson, E.S., 1991. An examination of anticipated g-gitter on the Space Station and its effects on materials processing. NASA TM 103775.

Rogers, M.J.B., DeLombard, R., 1995. Summary report of mission acceleration measurements for STS-66 launched November 3, 1994. NASA Technical Memorandum 106914, NASA Lewis Research Center, Cleveland.

Schlichting, H., 1932. Berechnung ebener periodischer Grenzschichtströmungen. Phys. Z. 33, $327-335$.

Simonenko, I.B., 1972. A justification of the averaging method for a problem of convection in a field rapidly oscillating forces and other parabolic equations. Mat. Sb. (129) 2, 245-263.

Simonenko, I.B., Zen'kovskaja, S.M., 1966. On the effect of high frequency vibrations on the origin of convection. Izv. Akad. Nauk SSSR Ser. Mech. Zhidk. Gaza 5, 51-55.

Terrones, G., Chen, C.F., 1993. Convective stability of gravity-modulated doubly cross-diffusive fluid layers. J. Fluid Mech. 255, 301-321.

Tsitverblit, N., 1995. Bifurcation phenomena in confined thermosolutal convection with lateral heating: Commencement of the double-diffusive region. Phys. Fluids 7, 718-736.

Viskanta, R., Bergman, T.L., Incropera, F.P., 1985. Double diffusive natural convection. In: Kaku, S., Aung, W., Viskanta, R. (Eds.), Natural convection: Fundamentals and Applications. Hemisphere, Washington, DC, pp. 1075-1099.

Wheeler, A.A., McFadden, G.B., Murray, B.T., Coriell, S.R., 1991. Convective stability for the Rayleigh-Bénard and directional solidification problems: high-frequency gravity modulation. Phys. Fluids 3, 2847-2858.

Wilcox, W.R., 1993. Transport phenomena in crystal growth from solution. Prod. Cryst. Growth Charact. $26,153-194$.

Xin, S., Le Quéré, P., Tuckerman, L., 1998. Bifurcation analysis of double-diffusive convection with opposing horizontal thermal and solutal gradients. Phys. Fluids 10, 850-858. 